\title{
84-GHz methanol masers, their relationship to 36-GHz methanol masers, and their molecular environments
}

\author{
S. L. Breen ${ }^{\oplus},{ }^{1 \star}$ Y. Contreras, ${ }^{2}$ J. R. Dawson, ${ }^{3}$ S. P. Ellingsen ${ }^{\oplus},{ }^{4}$ M. A. Voronkov ${ }^{5}$ and \\ T. P. McCarthy ${ }^{\oplus 4,5}$ \\ ${ }^{1}$ Sydney Institute for Astronomy (SIfA), School of Physics, University of Sydney, NSW 2006, Australia \\ ${ }^{2}$ Leiden Observatory, Leiden University, P.O. Box 9513, NL-2300 RA Leiden, the Netherlands \\ ${ }^{3}$ Department of Physics and Astronomy and MQ Research Centre in Astronomy, Astrophysics and Astrophotonics, Macquarie University, NSW 2109, Australia \\ ${ }^{4}$ School of Natural Sciences, University of Tasmania, Private Bag 37, Hobart, Tasmania 7001, Australia \\ ${ }^{5}$ CSIRO Astronomy and Space Science, Australia Telescope National Facility, PO Box 76, Epping, NSW 1710, Australia
}

Accepted 2018 December 11. Received 2018 November 30; in original form 2018 October 17

\begin{abstract}
We present observations of the $36-\mathrm{GHz}\left(4_{-1} \rightarrow 3_{0} E\right)$ and $84-\mathrm{GHz}\left(5_{-1} \rightarrow 4_{0} E\right)$ class I methanol maser transitions towards a sample of 94 known class I sites. These observations resulted in 93 and 92 detections in the 84- and 36-GHz transitions. While the majority of the $36-\mathrm{GHz}$ sources have been previously reported, many of the sites are observed in the $84-\mathrm{GHz}$ transition for the first time. The near-simultaneous observations of the two transitions revealed strikingly similar spectral profiles and a mean and median 36- to 84-GHz integrated flux density ratio of 2.6 and 1.4. Alongside the 36- and $84-\mathrm{GHz}$ observations, we included rare class II methanol masers at $37.7,38.3,38.5,86.6$, and $86.9 \mathrm{GHz}$, a number of recombination lines, and thermal molecular transitions. We detect one new site of 86.6- and 86.9-GHz methanol masers, as well as six maser candidates in one or more of 37.7-, 38.3-, 38.5-, 86.6-, and 86.9-GHz methanol maser transitions. We detect a relatively higher rate of $\mathrm{HC}_{3} \mathrm{~N}$ compared to that reported by MALT90 (once the respective detection limits were taken into account), who targeted dense dust clumps, suggesting that the class I methanol maser targets incorporate a relatively higher number of warm protostellar sources. We further find that there are similar relationships between the integrated flux density of both class I transitions with the integrated intensity of $\mathrm{HC}_{3} \mathrm{~N}, \mathrm{HNC}, \mathrm{HCO}^{+}, \mathrm{HNC}, \mathrm{SiO}$, and $\mathrm{H}^{13} \mathrm{CO}^{+}$. We suggest that this indicates that the integrated flux densities of the 36- and $84-\mathrm{GHz}$ transitions are closely linked to the available gas volume.
\end{abstract}

Key words: masers - stars: formation-ISM: molecules - radio lines: ISM.

\section{INTRODUCTION}

Methanol masers are important tracers of star formation, in part due to the complexity of the methanol molecule that results in numerous maser transitions, each prevalent within a slightly different range of physical conditions (e.g Cragg, Sobolev \& Godfrey 2005; McEwen, Pihlström \& Sjouwerman 2014; Leurini, Menten \& Walmsley 2016). The many transitions of methanol masers are empirically divided into two classes of sources (e.g. Batrla et al. 1987; Menten 1991b). Class I methanol masers are collisionally excited and tend to occupy the region surrounding an outflow or an expanding $\mathrm{H}$ II region (e.g. Voronkov et al. 2014), while class II methanol masers are radiatively pumped and so are generally much more closely

^E-mail: shari.breen@ sydney.edu.au associated with the young star formation region (e.g. Caswell et al. 2010). Whereas some class I methanol masers have been detected towards low-mass stars (e.g Kalenskii et al. 2010), class II methanol masers (at least at $6.7 \mathrm{GHz}$ ) are exclusively associated with highmass star formation regions (e.g. Minier et al. 2003; Xu et al. 2008; Breen et al. 2013).

In recent years, a definitive, unbiased search for class II methanol masers at $6.7 \mathrm{GHz}$ has been made in the Southern hemisphere (Caswell et al. 2010; Green et al. 2010; Caswell et al. 2011; Green et al. 2012; Breen et al. 2015), detecting 972 maser sites, each of which has been searched for accompanying 12.2-GHz emission, resulting in a detection rate of 45.3 per cent (Breen et al. 2012a,b, 2014, 2016). Other, rarer class II methanol maser observations are becoming more prevalent, and significant samples have now been targeted for the 19.9-, 23.1-, 37.7-, 38.3-, 38.5-, 85.5-, 86.6-, 86.9-, 107.0-, and 156.6-GHz transitions (e.g. Val'tts et al. 1999; 
Ellingsen et al. 2003, 2004; Cragg et al. 2004; Umemoto et al. 2007; Caswell et al. 2010; Ellingsen et al. 2011). These more rare transitions trace less commonly found physical conditions and their presence indicates either a short-lived evolutionary phase in the star formation process or unusual star formation regions. In some cases the rarer transitions can also be weaker (although high flux density sources also exist; e.g. Ellingsen et al. 2018), adding further complexity to their detection. Recently, studies of the transitions at $37.7,38.3$, and $38.5 \mathrm{GHz}$ have led to the suggestion that their short-lived presence may indicate the end of the class II methanol maser phase in the high-mass star formation process (Ellingsen et al. 2011, 2013) and the first high-resolution observations of these transitions have recently been made (Ellingsen et al. 2018). Other transitions, like the $86.6-\mathrm{GHz} 7_{2} \rightarrow 6_{3} A^{-}$and the $86.9-\mathrm{GHz} 7_{2}$ $\rightarrow 6_{3} A^{+}$transitions, are especially rare, even taking into account the relatively small number of searches that have been conducted. To date, maser emission in these transitions has only been detected towards G345.01+1.79, W3(OH), and W51-IRS1 (Cragg et al. 2001; Sutton et al. 2001; Minier \& Booth 2002; Ellingsen et al. 2003).

Class I methanol maser studies have also generally been limited to targeted observations (e.g. Kurtz, Hofner \& Álvarez 2004; Ellingsen 2005; Cyganowski et al. 2009; Chen et al. 2011; Voronkov et al. 2014; Gómez-Ruiz et al. 2016; Rodríguez-Garza et al. 2017), with the exception of a recently completed survey of 5 square degrees of the Southern Galaxy in the 44-GHz transition (Jordan et al. 2015, 2017), a small region towards the Galactic Centre in the 36$\mathrm{GHz}$ transition (Yusef-Zadeh et al. 2013), and a large-scale search for the rare 23.4-GHz transition (only one detection made in a relatively shallow search across a $100^{\circ} \times 1^{\circ}$ region of the Galactic plane; Voronkov et al. 2011). The most extensively studied lines are those at 36, 44, and $95 \mathrm{GHz}$ and have resulted in hundreds of detections across the Galaxy (e.g. Chen et al. 2011; Voronkov et al. 2014; Jordan et al. 2017). The $5_{-1} \rightarrow 4_{0} E$ class I methanol maser transition at $84 \mathrm{GHz}$ is particularly poorly characterized, having only been observed in a small number of searches since the transition was first detected towards DR 21(OH), NGC 2264, and OMC-2 (Batrla \& Menten 1988; Menten 1991a). The most extensive search for this transition was conducted by Kalenskii et al. (2001), who targeted 51 class I methanol masers, detecting narrow maser-like emission towards 14 of their targets and quasi-thermal emission towards a further 34. An additional search by Rodríguez-Garza et al. (2018) targeted 38 sites of 44-GHz methanol maser emission with the Large Millimeter Telescope and resulted in a detection rate of 74 per cent despite a velocity resolution of $\sim 100 \mathrm{~km} \mathrm{~s}^{-1}$. Further, targeted observations of this transition have included a limited number of sources, revealing detections at the locations of both high- and low-mass star formation regions (e.g. Salii, Sobolev \& Kalinina 2002; Kalenskii et al. 2006). Maser emission from the 84-GHz transition $\left(5_{-1} \rightarrow 4_{0} E\right)$ is expected to be similar to the more widely studied $36-\mathrm{GHz}\left(4_{-1} \rightarrow 3_{0} E\right)$ transition given that they are consecutive transitions in the same transition series. The more commonly observed class I masers at $44\left(7_{0} \rightarrow 6_{1} A^{+}\right)$and $95 \mathrm{GHz}\left(8_{0} \rightarrow 7_{1} A^{+}\right)$are also consecutive transitions in the same $(J+1)_{0} \rightarrow J_{1} A^{+}$transition series. The $95-\mathrm{GHz}$ transition has a high detection rate towards $44-\mathrm{GHz}$ sources, but is usually about a factor of 3 weaker (e.g Val'tts 2000; McCarthy et al. 2018a).

Here we present a series of spectral line observations conducted with the Mopra radio telescope towards 94 class I methanol maser targets (Kurtz et al. 2004; Voronkov et al. 2014). The primary goal of the observations was to detect new sources of the poorly studied 84-GHz class I methanol maser transition, with quasi-simultaneous observations of the $36-\mathrm{GHz}$ class I methanol maser line also included to obtain meaningful line ratios to inform maser pumping models and to allow comparisons with observations of extragalactic class I sources (e.g. Ellingsen et al. 2017; McCarthy et al. 2017; McCarthy et al. 2018b). Alongside these main target lines, we were able to include a number of other, rare class II methanol maser lines (at 37.7-, 38.3-, 38.5-, 86.6-, and 86.9-GHz) as well as a number of recombination lines and thermal lines, tracing dense and shocked gas, and allowing us to make some comparisons between the detected methanol masers and their environments. Given that the Mopra beam is $1.3 \mathrm{arcmin}$ at $36 \mathrm{GHz}$ and $0.6 \operatorname{arcmin}$ at $84 \mathrm{GHz}$, on the scale of whole clumps rather than individual star formation regions, we present the results and discussion with this in mind.

\section{OBSERVATIONS AND DATA REDUCTION}

\subsection{Targets}

Our observations targeted the locations of 94 known sites of class I methanol maser emission, comprising the full Voronkov et al. (2014) sample of 71 southern 36- and 44-GHz class I methanol masers along with a further 23 sources from the Kurtz et al. (2004) sample of 44-GHz class I methanol masers (we excluded 14 sources from their sample as their declinations were north of +20 degrees).

Voronkov et al. (2014) targeted their 36- and 44-GHz class I methanol maser observations towards known class I methanol masers south of a declination of -35 degrees, combining detections reported by Slysh et al. (1994), Val'tts (2000) and Ellingsen (2005), which themselves targeted the locations of $\mathrm{H}$ II regions, $6.7-\mathrm{GHz}$ methanol maser emission and $22-\mathrm{GHz}$ water maser emission. Kurtz et al. (2004) directed their 44-GHz class I methanol maser observations towards a diverse sample of 44 star formation regions, including both very young regions devoid of accompanying UCH II regions as well as those slightly more evolved sources with developed UCH II regions. Our sample of 94 targets therefore represents a range of sources, initially detected in a range of selection methods, and as such should not be dominated by a particular class of objects.

Both Voronkov et al. (2014) and Kurtz et al. (2004) provide spot maps of each of their sources, revealing the extent of the class I methanol maser emission in each case. We have targeted the centre of the class I maser emission region as reported by Voronkov et al. (2014) for 71 of our targets and observed the averaged right ascension and declinations of the maser spot positions reported in Kurtz et al. (2004) for the remaining 23. Our full target list, together with appropriate references, is given in Table 2.

\subsection{Observations}

We conducted targeted observations at both 7 and $3 \mathrm{~mm}$ towards the 94 class I methanol maser sites, using the Mopra 22-m radio telescope between 2018 April 30 and 2018 May 6. At both frequencies, the Mopra spectrometer (MOPS) was configured to record two orthogonal linear polarizations across 16 sub-bands, each covering $138 \mathrm{MHz}$ with 4096 channels. During the $7 \mathrm{~mm}$ observations, these sub-bands were distributed in the frequency range of 33077 to $40805 \mathrm{MHz}$ and during the $3 \mathrm{~mm}$ observations between 84468 and $92127 \mathrm{MHz}$, allowing us to observe up to 16 lines simultaneously at both 7 and $3 \mathrm{~mm}$. This configuration resulted in a velocity coverage of $\sim 1100 \mathrm{~km} \mathrm{~s}^{-1}$ and a native velocity resolution of $\sim 0.34 \mathrm{~km} \mathrm{~s}^{-1}$ at $36.2-\mathrm{GHz}$ and a velocity coverage of $480 \mathrm{~km} \mathrm{~s}^{-1}$ and a native velocity resolution of $\sim 0.14 \mathrm{~km} \mathrm{~s}^{-1}$. The targeted lines are listed in Table 1 , and includes the typical $1 \sigma$ noise limits as well as the final 
Table 1. Target spectral lines split into methanol masers (top), thermal molecular lines (middle), and radio recombination lines (bottom). The observed line is followed by the adopted rest frequency (with uncertainties listed for the maser transitions in units of the least significant figure), the velocity resolution (post-processing), the $\mathrm{K}$ to Jy conversion factor used (where appropriate), the typical $1 \sigma$ noise level (in Jy where a conversion factor is given otherwise in $\mathrm{K}$ ), the rest frequency reference, and finally notes indicating the class of maser or drawing attention to lines that had an incomplete coverage in our sample due to a sub-optimal frequency set-up ('Incomplete') and are therefore not observed for every source.

\begin{tabular}{|c|c|c|c|c|c|c|}
\hline Spectral line & $\begin{array}{l}\text { Rest } \\
\text { frequency } \\
(\mathrm{MHz})\end{array}$ & $\begin{array}{l}V_{\text {res. }} \\
\left(\mathrm{km} \mathrm{s}^{-1}\right)\end{array}$ & $\begin{array}{l}\mathrm{K} \text { to Jy } \\
\text { factor }\end{array}$ & $\begin{array}{l}\text { Typical } \\
\text { noise } \\
\text { (Jy or K) }\end{array}$ & Reference & Notes \\
\hline $\mathrm{CH}_{3} \mathrm{OH} 7_{-2} \rightarrow 8_{-1} E$ & $37703.696(13)$ & 0.32 & 14 & 0.8 & Xu \& Lovas (1997) & Class II \\
\hline $\mathrm{CH}_{3} \mathrm{OH} 6_{2} \rightarrow 5_{3} A^{-}$ & $38293.292(14)$ & 0.32 & 14 & 0.8 & Xu \& Lovas (1997) & Class II \\
\hline $\mathrm{CH}_{3} \mathrm{OH} 6_{2} \rightarrow 5_{3} A^{+}$ & $38452.652(14)$ & 0.32 & 14 & 0.8 & Xu \& Lovas (1997) & Class II \\
\hline $\mathrm{CH}_{3} \mathrm{OH} 5_{-1} \rightarrow 4_{0} E$ & $84521.169(10)$ & 0.20 & 16 & 0.8 & Müller, Menten \& Mäder (2004) & Class I \\
\hline $\mathrm{CH}_{3} \mathrm{OH} 7_{2} \rightarrow 6_{3} A^{+}$ & $86902.949(5)$ & 0.20 & 16 & 0.8 & Müller et al. (2004) & Class II \\
\hline $\mathrm{H}^{13} \mathrm{CN}$ & 86339.9214 & 0.20 & - & 0.04 & Remijan et al. (2007) & Incomplete \\
\hline $\mathrm{H}^{13} \mathrm{CO}^{+}(1-0)$ & 86754.2884 & 0.20 & - & 0.04 & Remijan et al. (2007) & \\
\hline $\mathrm{SiO}(2-0) v=0$ & 86846.96 & 0.20 & - & 0.04 & Remijan et al. (2007) & \\
\hline $\operatorname{HCN}(1-0)$ & 88631.847 & 0.19 & - & 0.04 & Remijan et al. (2007) & \\
\hline $\mathrm{CH}_{3} \mathrm{OH} 15_{3} \rightarrow 14_{4} A^{-}$ & 88940.09 & 0.19 & - & 0.04 & Remijan et al. (2007) & \\
\hline $\mathrm{CH}_{3} \mathrm{CN} 5(1)-4(1)$ & 91985.3141 & 0.19 & - & 0.05 & Remijan et al. (2007) & \\
\hline $\mathrm{H} 72 \beta$ & 33821.51 & 0.51 & - & 0.04 & Lilley \& Palmer (1968) & \\
\hline $\mathrm{H} 57 \alpha$ & 34596.39 & 0.50 & - & 0.04 & Lilley \& Palmer (1968) & \\
\hline $\mathrm{H} 69 \beta$ & 38360.28 & 0.48 & - & 0.04 & Lilley \& Palmer (1968) & \\
\hline $\mathrm{H} 55 \alpha$ & 38473.36 & 0.45 & - & 0.04 & Lilley \& Palmer (1968) & \\
\hline $\mathrm{H} 42 \alpha$ & 85688.40 & 0.20 & - & 0.06 & Lilley \& Palmer (1968) & Incomplete \\
\hline $\mathrm{H} 41 \alpha$ & 92034.45 & 0.19 & - & 0.06 & Lilley \& Palmer (1968) & \\
\hline
\end{tabular}

velocity resolution (taking any smoothing into account).

At both frequency set-ups, the pointing was corrected by observing a nearby $\mathrm{SiO}$ maser approximately once per hour, resulting in pointing uncertainties of less than 10 arcsec. Each target was observed in a series of position-switched observations, with reference observations made -15 arcmin in declination from each target. At $7 \mathrm{~mm}$ we observed a reference, source, source, reference pattern once and spent $1 \mathrm{~min}$ at each position, which resulted in a total on-source integration time of $2 \mathrm{~min}$. At $3 \mathrm{~mm}$ we repeated the same observation pattern twice, and spent $2 \mathrm{~min}$ at each position, making our total on-source integration time $8 \mathrm{~min}$. At $7 \mathrm{~mm}$ the system temperature was measured solely by a continuously switched noise diode, but at $3 \mathrm{~mm}$ we also made paddle measurements every 15 $20 \mathrm{~min}$ to achieve a calibrated antenna temperature. We estimate that the flux density/antenna temperature measurements are accurate to 20 percent (taking into account residual pointing errors, opacity variations, and primary calibration errors).

At the frequency of the $36-\mathrm{GHz}$ methanol maser line, the halfpower beam width (HPBW) of Mopra is $1.3 \mathrm{arcmin}$ and at the frequency of the 84-GHz methanol maser line it is 0.6 arcmin, which are both large enough to accommodate the majority of expected class I methanol maser distributions (Voronkov et al. 2014).

\subsection{Data reduction}

The data were processed using the ATNF Spectral Analysis Package (ASAP) using standard techniques for position-switched observations. Alignment of the velocity channels was carried out during processing and the adopted rest frequencies are given in Table 1. For some of the lines, data were Hanning-smoothed during the processing and the resultant velocity resolutions are also given in Table 1. The maser data were converted from antenna temperature to Janskys following Urquhart et al. (2010) at $7 \mathrm{~mm}$, who give conversion factors of 13.6 and 14 for the $36-\mathrm{GHz}$ methanol transition and the three class II transitions near $38 \mathrm{GHz}$, respectively. At $3 \mathrm{~mm}$ the conversion factor was calculated using the main beam efficiency presented by Ladd et al. (2005), which implies an antenna temperature to Jansky conversion factor of 16 . For each of the maser transitions the conversion factor used is listed in Table 1. Molecular and recombination lines are presented in units of antenna temperature $\left(T_{A}^{*}\right)$.

Typical $1 \sigma$ noise limits for each of the lines are given in Table 1 in units of $\mathrm{Jy}$ for the masers and antenna temperature for all other lines. In the case where $36-$ and $84-\mathrm{GHz}$ emission is not detected, source-specific $3 \sigma$ detection limits are given in Table 2 and likewise for the thermal molecular and recombination lines listed in Table A1. In the case of the molecular and recombination lines, we fitted Gaussian profiles to the emission. In the simple cases for lines without hyperfine structure, a single Gaussian component was used to determine the peak antenna temperature, peak velocity, line width, and integrated intensity. For HCN we simultaneously fitted the hyperfine components and have presented the peak antenna temperature, peak velocity, line width of the main line, and the combined integrated intensity, including the hyperfine components. In the case of $\mathrm{CH}_{3} \mathrm{CN}$ we have fitted each of the detected hyperfine components simultaneously but presented each 
Table 2. 36- and 84-GHz methanol detections towards class I methanol maser sites reported in Voronkov et al. (2014) and Kurtz et al. (2004). Source names (column 1) have been adopted from those publications, but where those names do not represent the Galactic coordinates, they are presented in parentheses following the adopted name. Columns 2 and 3 give the targeted right ascension and declination (J2000), followed by two groups of five columns which give the minimum, maximum, and peak velocity, the peak flux density, and the integrated flux density for the 36- and 84-GHz transitions (in units of Jy km s ${ }^{-1}$ ), respectively. In the case where transitions are not detected, $3 \sigma$ detection limits are given in place of a measured peak flux density. Targets from the Voronkov et al. (2014) sample were observed at $36 \mathrm{GHz}$ in that work and references to previous detections of 84-GHz methanol emission are 1: Kalenskii et al. (2001); 2: Voronkov et al. (2006).

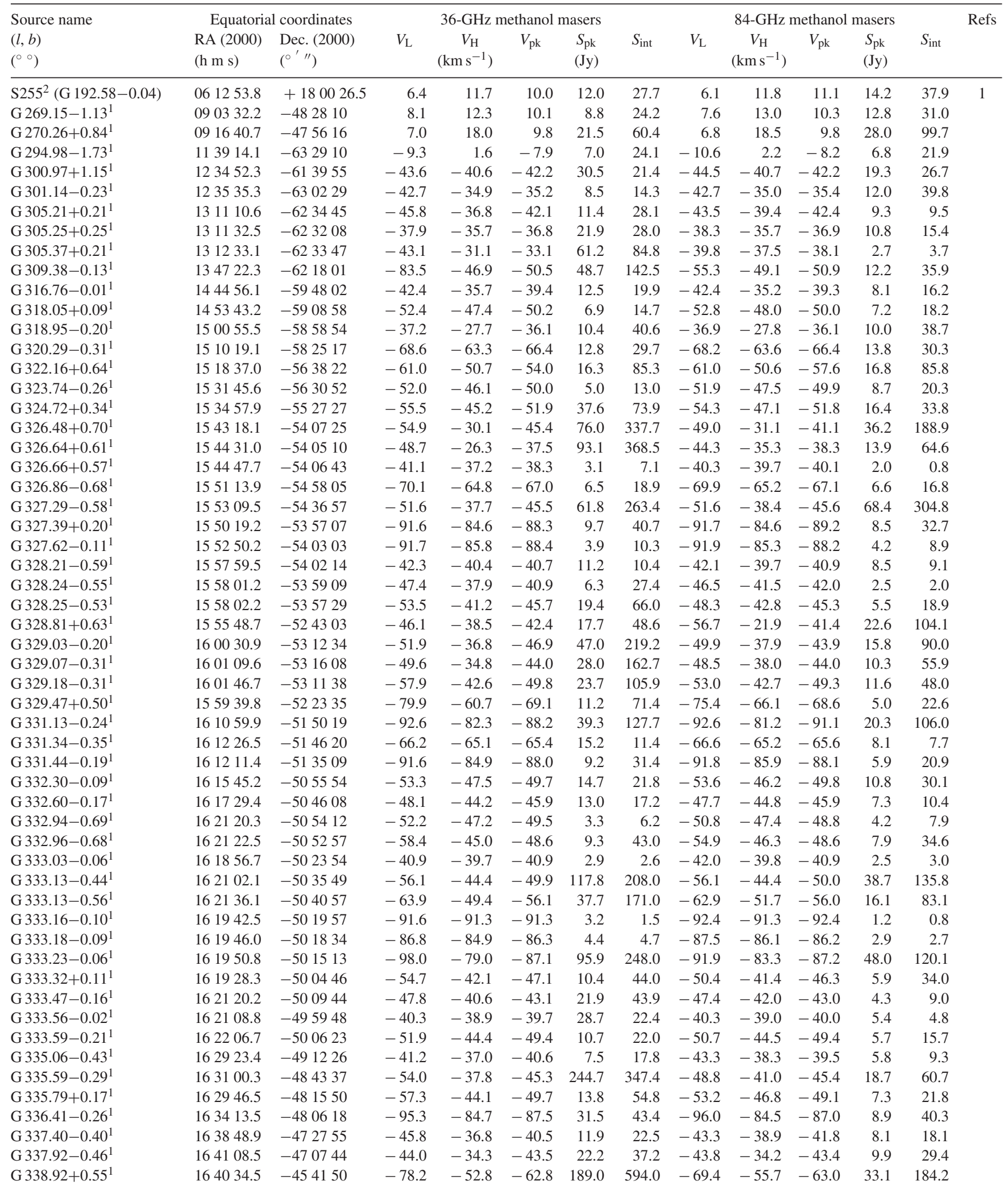


Table 2 - continued

\begin{tabular}{|c|c|c|c|c|c|c|c|c|c|c|c|c|c|}
\hline \multirow{2}{*}{$\begin{array}{l}\text { Source name } \\
(l, b) \\
\left({ }^{\circ}\right)\end{array}$} & \multicolumn{2}{|c|}{ Equatorial coordinates } & \multicolumn{5}{|c|}{ 36-GHz methanol masers } & \multicolumn{5}{|c|}{ 84-GHz methanol masers } & \multirow[t]{2}{*}{ Refs } \\
\hline & $\begin{array}{l}\text { RA (2000) } \\
(\mathrm{h} \mathrm{m} \mathrm{s})\end{array}$ & $\begin{array}{l}\text { Dec. (2000) } \\
\left(0^{\prime}{ }^{\prime \prime}\right)\end{array}$ & $V_{\mathrm{L}}$ & $\begin{array}{c}V_{\mathrm{H}} \\
\left(\mathrm{km} \mathrm{s}^{-1}\right)\end{array}$ & $V_{\mathrm{pk}}$ & $\begin{array}{l}S_{\mathrm{pk}} \\
(\mathrm{Jy})\end{array}$ & $S_{\text {int }}$ & $V_{\mathrm{L}}$ & $\begin{array}{c}V_{\mathrm{H}} \\
\left(\mathrm{km} \mathrm{s}^{-1}\right)\end{array}$ & $V_{\mathrm{pk}}$ & $\begin{array}{l}S_{\mathrm{pk}} \\
(\mathrm{Jy})\end{array}$ & $S_{\text {int }}$ & \\
\hline $\mathrm{G} 341.19-0.23^{1}$ & 165216.4 & -442844 & -63.5 & -15.2 & -41.7 & 83.6 & 122.8 & -51.3 & -41.7 & -41.8 & 7.1 & 3.2 & \\
\hline G $341.22-0.21^{1}$ & 165217.4 & -442703 & -46.2 & -38.9 & -43.1 & 25.6 & 56.1 & -48.2 & -39.0 & -43.2 & 15.0 & 41.7 & \\
\hline $\mathrm{G} 343.12-0.06^{1}$ & 165816.8 & -425209 & -36.6 & -22.9 & -27.1 & 43.3 & 115.7 & -36.5 & -24.1 & -27.3 & 23.4 & 79.6 & 2 \\
\hline G $345.01+1.79^{1}$ & 165646.0 & -401409 & -19.6 & -9.9 & -13.2 & 55.7 & 111.6 & -20.1 & -9.1 & -13.2 & 46.8 & 135.5 & \\
\hline G $345.42-0.95^{1}$ & 170935.6 & -413540 & -24.8 & -17.5 & -17.8 & 4.0 & 8.9 & -23.8 & -21.3 & -23.0 & 2.4 & 3.4 & \\
\hline $\mathrm{G} 345.50+0.35^{1}$ & 170424.1 & -404407 & -19.9 & -13.5 & -17.4 & 6.1 & 25.7 & -19.8 & -14.0 & -17.4 & 3.2 & 12.0 & \\
\hline $\mathrm{G} 348.18+0.48^{1}$ & 171206.8 & -383038 & -10.0 & -1.3 & -7.2 & 37.1 & 82.1 & -9.5 & -2.8 & -6.8 & 8.9 & 30.8 & \\
\hline G $349.09+0.11^{1}$ & 171624.6 & -375943 & -82.0 & -73.0 & -78.1 & 21.4 & 41.9 & -81.9 & -73.3 & -78.1 & 15.4 & 36.3 & \\
\hline G $351.16+0.70^{1}$ & 171956.4 & -355754 & -11.4 & -1.6 & -6.1 & 67.4 & 185.4 & -11.8 & -2.3 & -6.3 & 27.8 & 101.3 & \\
\hline $\mathrm{G} 9.62+0.19^{2}$ & 180615.1 & -203137.1 & 2.2 & 7.5 & 3.8 & 7.1 & 22.5 & 2.1 & 7.4 & 3.4 & 6.3 & 23.5 & \\
\hline $\mathrm{G} 10.47+0.03^{2}$ & 180837.9 & -195134.2 & 61.1 & 74.6 & 68.1 & 12.3 & 79.8 & 60.2 & 73.0 & 66.0 & 7.9 & 39.9 & \\
\hline G $10.6-0.4^{2}$ & 181029.0 & -195546.4 & -9.1 & 2.1 & -5.7 & 49.1 & 106.5 & -9.3 & 2.2 & -6.6 & 9.3 & 51.1 & \\
\hline $\mathrm{GGD}^{2} 7^{2}(\mathrm{G} 10.84-2.59)$ & 181912.4 & -204724.8 & 11.1 & 25.9 & 13.4 & 39.8 & 39.2 & 11.2 & 14.5 & 13.1 & 29.1 & 27.7 & \\
\hline G $11.94-0.62^{2}$ & 181402.2 & -185332.0 & 32.7 & 40.6 & 38.3 & 5.7 & 23.4 & 35.1 & 39.4 & 38.1 & 3.4 & 7.6 & \\
\hline $\mathrm{G} 12.21-0.10^{2}$ & 181240.1 & -182421.4 & 18.5 & 30.5 & 23.8 & 6.6 & 43.8 & 18.2 & 29.3 & 23.7 & 6.2 & 43.8 & \\
\hline G $12.89+0.49^{2}$ & 181150.8 & -173135.9 & 31.2 & 36.3 & 31.5 & 10.3 & 13.3 & 31.0 & 35.6 & 31.5 & 4.5 & 10.5 & \\
\hline $\mathrm{Mol}^{2} 5^{2}$ (G 13.66-0.60) & 181723.6 & -172213.0 & 42.6 & 53.7 & 48.7 & 30.8 & 64.1 & 43.7 & 49.6 & 48.4 & 11.7 & 28.1 & \\
\hline $\mathrm{Mol}^{2} 0^{2}$ (G 14.89-0.40) & 181907.6 & -161125.6 & 61.4 & 64.4 & 62.2 & 24.2 & 23.8 & 61.2 & 64.4 & 62.2 & 5.3 & 6.7 & \\
\hline G $19.62-0.23^{2}$ & 182737.7 & -115636.5 & 36.5 & 46.3 & 41.3 & 9.0 & 16.0 & 37.8 & 45.9 & 41.1 & 7.2 & 15.9 & 1 \\
\hline G $29.96-0.02^{2}$ & 184603.1 & -023926.2 & 95.2 & 101.6 & 98.0 & 3.6 & 11.8 & 95.6 & 99.5 & 97.5 & 3.1 & 7.5 & 1 \\
\hline $\mathrm{G} 31.41+0.31^{2}$ & 184734.4 & -011248.8 & 93.4 & 101.5 & 98.4 & 12.4 & 47.6 & 92.3 & 102.1 & 98.5 & 8.6 & 47.9 & 1 \\
\hline W51Nc ${ }^{2}(\mathrm{G} 49.49-0.37)$ & 192340.1 & +143113.8 & 47.9 & 71.7 & 59.9 & 10.5 & 95.4 & 52.8 & 66.0 & 62.4 & 3.3 & 18.3 & \\
\hline
\end{tabular}

of them individually. Given our limited signal-to-noise ratio, we have detected a range in the number of components from one right through to five.

\section{RESULTS}

Observations of 7 different methanol maser transitions, 10 molecular lines, and 6 recombination lines have resulted in a rich data set, with many detections of the target lines. Given the large number of lines, we describe the results in sub-groups. For some sources that require additional information to that which can be described in tables and spectra there are comments given in Section 3.5.

\subsection{6- and 84-GHz methanol sources}

Of the 94 class I methanol masers targeted (previously characterized at either or both of the 36- and 44-GHz transitions), we have detected 92 in the $36-\mathrm{GHz}$ transition and 93 in the $84-\mathrm{GHz}$ transition. The two sources we failed to detect at $36 \mathrm{GHz}$ were Mol77 and G 45.07+0.13, which were both reported by Kurtz et al. (2004) as
44-GHz class I methanol masers (Mol77 is also the only source where no emission was detected in the $84-\mathrm{GHz}$ methanol line). The first of these was detected with a peak flux density of $0.57 \mathrm{Jy}$ and the second was detected at $1.08 \mathrm{Jy}$ by Kurtz et al. (2004) during their observations in 1999 and 2000, respectively. Given that 44$\mathrm{GHz}$ masers are generally stronger than accompanying emission in the 36-GHz transition (Voronkov et al. 2014), and that our $3 \sigma$ $36-\mathrm{GHz}$ detection limits are higher than the reported $44-\mathrm{GHz}$ peak flux densities (1.9 and $1.8 \mathrm{Jy}$ for the 36- and $84-\mathrm{GHz}$ transitions in Mol77 and 2.9 Jy for the 36-GHz transition in $\mathrm{G} 45.07+0.13$ ), their non-detection is expected.

The properties of both the 36- and 84-GHz masers are given in Table 2, including $3 \sigma$ detection limits where appropriate. References to previously detected $84-\mathrm{GHz}$ sources are given in the final column, indicating that only 6 of the 93 detections have been reported in the literature previously. References to previously detected $36-\mathrm{GHz}$ masers are not explicitly given in Table 2 but those 71 targets taken from Voronkov et al. (2014) (indicated by a ${ }^{~} 1$ ' following the source name) were characterized at both 36 and $44 \mathrm{GHz}$ in that work. The remaining 23 targets are taken from a $44-\mathrm{GHz}$ methanol maser 
catalogue (Kurtz et al. 2004) for which few sources have been followed up at $36 \mathrm{GHz}$ previously.

For each of the 94 class I methanol maser targets, spectra of the detected $84-\mathrm{GHz}$ sources have been overlaid with the detected 36-GHz emission in Fig. 1. For completeness we have included the three spectra where we fail to detect any emission. These spectra highlight that the structure of the two transitions is remarkably similar in almost all cases.

\subsection{7-, 38.3-, and 38.5-GHz methanol masers}

Alongside our observations of class I 36-GHz methanol masers, we were able to simultaneously observe the rarer, 37.7-, 38.3-, and $38.5-\mathrm{GHz}$ class II methanol maser lines. Despite the $7 \mathrm{~mm}$ observations being motivated by the (generally) much stronger 36$\mathrm{GHz}$ transition, which required very short on-source integration times $(2 \mathrm{~min})$, we were able to detect emission from a number of maser lines. In total we detect seven known 37.7-GHz masers, three known 38.3-GHz masers, and two known 38.5-GHz masers (Haschick, Baan \& Menten 1989; Ellingsen et al. 2011, 2013, 2018). While these known masers account for most of our detections, we also present a further six maser candidates that are very close to the $3 \sigma$ detection limit of the observations. While their peak flux densities are low, they all have velocities close to both the detected 36-GHz class I methanol maser emission and the velocity ranges of reported 6.7-GHz methanol maser emission (Green et al. 2010; Caswell et al. 2011; Green et al. 2012). The properties of the previously detected sources, together with our six maser candidates, are presented in Table 3. Spectra for each of the listed sources are given in Fig. 2.

Of the six maser candidates, three are accounted for by G 327.29-0.58, which shows very weak potential emission in each of the three transitions. If these transitions were considered in isolation, the 'emission' would not be notable, but given the marginal detections at the same velocity in each of the transitions it is worth including in more sensitive follow-up observations. Further discussion of this source, together with the other marginal detections, is presented in Section 3.5.

\subsection{6- and 86.9-GHz methanol detections}

Towards the 94 target class I methanol maser sites we detect nine sites exhibiting 86.6- and 86.9-GHz emission. Spectra of each of these detections are presented in Fig. 3 and their properties are given in Table 4. Of the nine detections, four appear to have typically thermal spectral profiles (G 327.29-0.58, G 351.77-0.54, $\mathrm{G} 34.26+0.15$, and W51E1), three have some indications of narrow maser-like emission (G339.88-1.26, G 344.23-0.57, and $\mathrm{G} 351.42+0.65)$, and two appear to be maser emission (G 345.01+1.79 and G 29.96-0.02). As indicated in Table 4 five of these are new detections in these transitions, including one of the maser sources and two of the possible maser candidates.

Five of the nine detections also show emission (or potential emission) in one or more of the 37.7-, 38.3-, or 38.5-GHz transitions. These sources are discussed further in Section 3.5.

\subsection{Molecular and recombination line detections}

During our targeted methanol maser observations, we were able to simultaneously observe a number of molecular and radio recombination lines. The parameters of these lines, derived from Gaussian fitting, are presented in Table 1 . In the case where no emission is detected a $3 \sigma$ detection limit is given. We only list $\mathrm{H}^{13} \mathrm{CN}$ and $\mathrm{H} 42 \alpha$ when the velocity range of the given source is included in the observations.

The detection rates of each of the lines that have a reasonably complete set of observations (i.e. the observing bandwidth accommodated the full complement of velocity ranges, so excludes $\mathrm{H}^{13} \mathrm{CN}$ ) are presented in Fig. 4, showing that we detect $\mathrm{HNC}, \mathrm{HCN}$, $\mathrm{HCO}^{+}, \mathrm{H}^{13} \mathrm{CO}^{+}$, and $\mathrm{HC}_{3} \mathrm{~N}$ towards 94.7 per cent of the methanol maser targets. $\mathrm{SiO}$ emission was detected towards 80.9 per cent of our target sources. $\mathrm{CH}_{3} \mathrm{CN}$ was detected towards 69.1 percent of the targets, although often not the full complement of hyperfine components, preventing us from deriving temperatures. Thermal methanol transitions $15_{3} \rightarrow 14_{4} A^{-}$and $8_{-4} \rightarrow 9_{-3} E$ are detected towards just 9 and 8 of the targets, respectively. One or more of the radio recombination lines were detected towards 29.8 percent of the sample.

\subsection{Comments on individual sources}

In this section we draw attention to notable sources, associations, marginal detections, and other details that are not able to be described fully in the source tables and spectra.

S255 (G 192.58-0.04). Kurtz et al. (2004) detected 44-GHz class I methanol maser emission towards this source using the Very Large Array (VLA). Pratap et al. (2008) conducted further single-dish observations of the 44-GHz emission in this source and used their derived position for targeted $36-\mathrm{GHz}$ methanol maser emission. Their position was 49 arcsec offset from the Kurtz et al. (2004) position, comparable to their HPBW, likely accounting for their nondetection in the 36-GHz transition. We detected 36-GHz methanol maser emission with a peak flux density of $12 \mathrm{Jy}$ at the Kurtz et al. (2004) 44-GHz methanol maser position.

G300.97+1.15. Although the candidate $37.7-\mathrm{GHz}$ methanol maser emission we detect towards this source is relatively weak ( $2.1 \mathrm{Jy}$ ), its velocity is coincident with the $36-\mathrm{GHz}$ methanol maser emission. Ellingsen et al. (2011) targeted this site for $37.7-\mathrm{GHz}$ methanol maser emission previously, achieving a $3 \sigma$ detection limit of $5.7 \mathrm{Jy}$, significantly higher than the emission detected in the current observations.

G 318.95-0.20. We detect a 37.7-GHz methanol maser towards this site, with a similar peak flux density to previous observations (Ellingsen et al. 2013, 2018). While we failed to detect any emission from the 38.3- and 38.5-GHz transitions, Ellingsen et al. (2018) report detections of these transitions with peak flux densities of 0.14 and $0.12 \mathrm{Jy}$, well below our $3 \sigma$ detection limits.

G 323.74-0.26. This site has been detected in the 37.7-GHz transition by Ellingsen et al. (2011, 2013, 2018), exhibiting some temporal variability between the observation epochs. The 2011 observations of Ellingsen et al. (2018) found a peak flux density of $16.1 \mathrm{Jy}$, while the current observations detect emission of $32 \mathrm{Jy}$. The higher sensitivity observations of Ellingsen et al. (2018) also allowed the detection of weak emission $(0.28$ and $0.22 \mathrm{Jy})$ in the 38.3- and 38.5-GHz transitions.

G 327.29-0.58. Very marginal emission is detected towards this source at $37.7,38.3$, and $38.5 \mathrm{GHz}$ as well as more significant thermal emission in the 86.6- and 86.9-GHz transitions. This is also one of the nine sources where the 88.9- and $89.5-\mathrm{GHz}$ methanol transitions are detected. The fact that emission is detected in all the transitions makes the marginal emission in the 37.7-, 38.3-, and 38.5-GHz lines believable. 

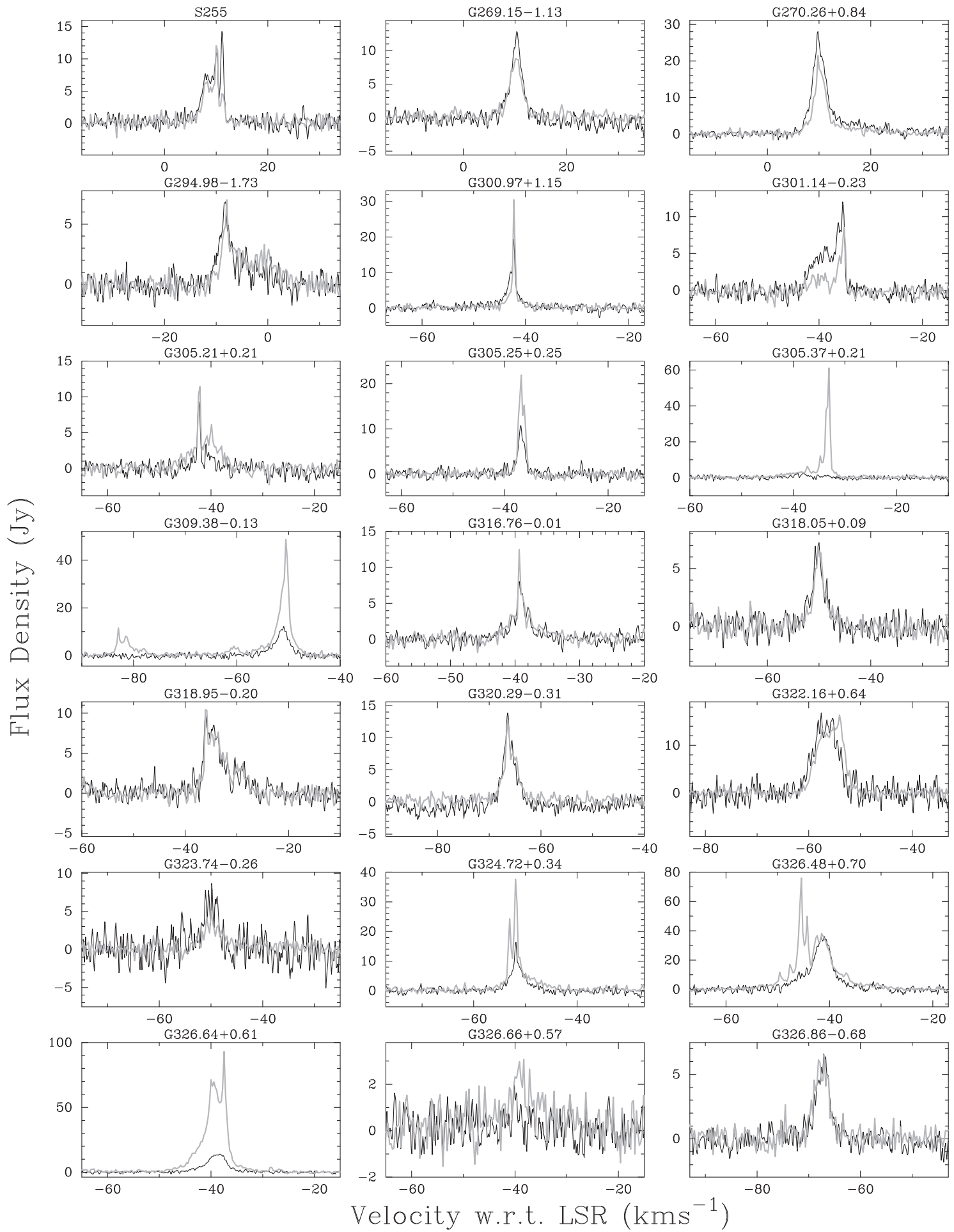

Figure 1. Spectra of the 84-GHz methanol (black) and 36-GHz methanol (grey) sources detected towards class I methanol maser targets.

G 335.79+0.17. Both the 37.7- and 38.3-GHz masers we detect have been reported previously by Ellingsen et al. (2013) with peak flux densities of 13.8 and $7 \mathrm{Jy}$, respectively. We detect emission with the same peak velocity $\left(\sim 46.1 \mathrm{~km} \mathrm{~s}^{-1}\right)$ but with peak flux densities of 8.2 and $4.7 \mathrm{Jy}$, respectively. The target positions of the Ellingsen et al. (2013) observations are within a couple of arcseconds (so within the pointing uncertainty of the Mopra telescope) of the position we targeted, so a pointing offset cannot account for the difference. Ellingsen et al. (2013) commented that this source was unusual as the only source that has been found to have emission in the $38.3-\mathrm{GHz}$ transition but not in the $38.5-\mathrm{GHz}$ transition.

G339.88-1.26. This site hosts the 37.7-GHz methanol maser with the highest flux density: $400 \mathrm{Jy}$ in our observations. With their 

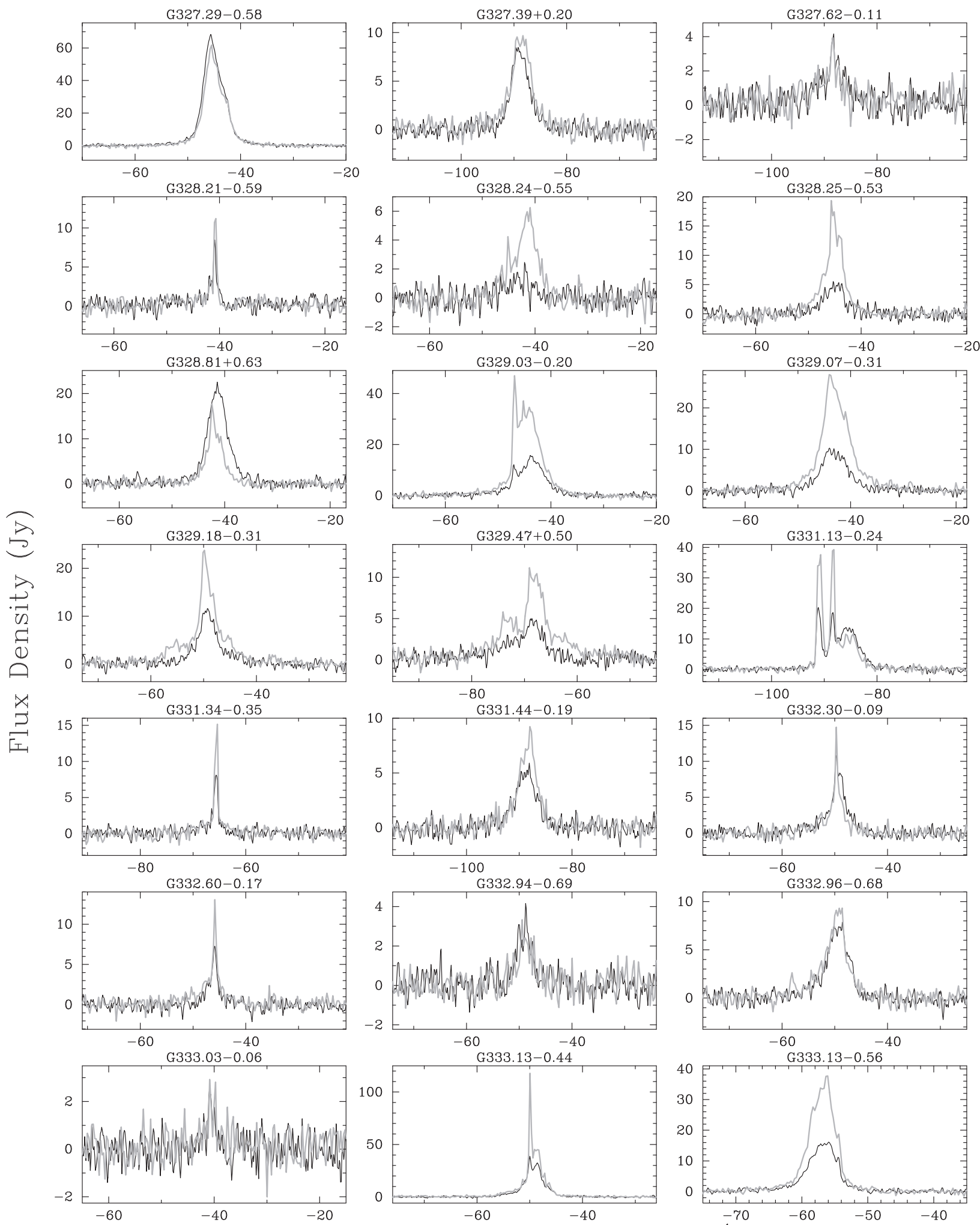

Velocity w.r.t. LSR $\left(\mathrm{kms}^{-1}\right)$

Figure 1. continued 

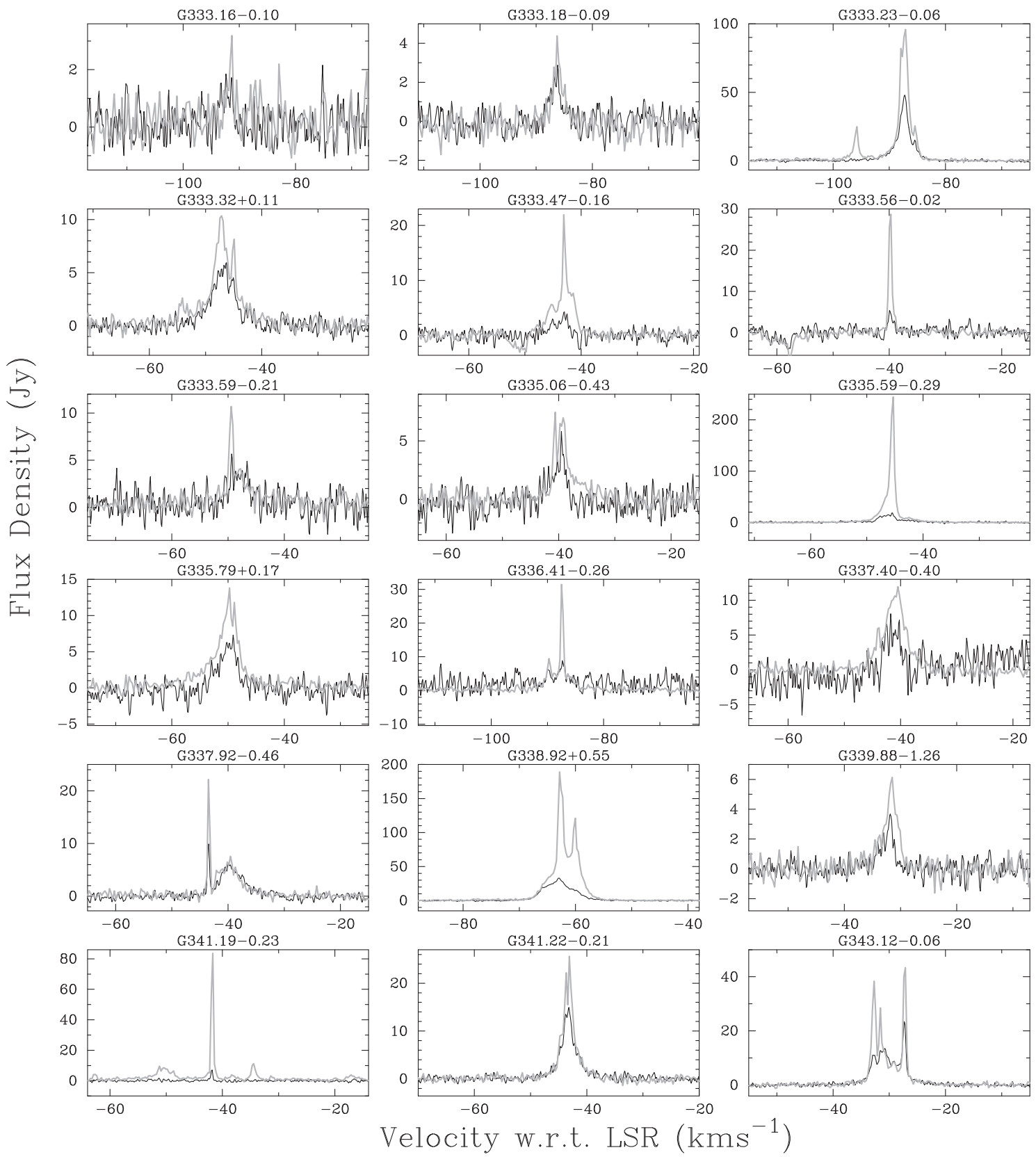

Figure 1. continued

higher sensitivity observations, Ellingsen et al. (2018) also detect emission in the 38.3- and $38.5-\mathrm{GHz}$ transitions, with peak flux densities of 0.93 and $0.69 \mathrm{Jy}$.

In the $86.6-\mathrm{GHz}$ transition we detect very marginal emission, which is only reportable given the presence of slightly stronger 86.9-GHz emission, together with the previously reported tentative detection made at this site by Ellingsen et al. (2003). The current observations are not of sufficient sensitivity for us to be able to definitively rule out that some of the emission may be arising from a maser. Further, sensitive observations will be required to confidently infer its nature. We detect no emission from the 88.9 - and $89.5-\mathrm{GHz}$ methanol transition.

G 343.12-0.06. Voronkov et al. (2006) conducted high spatial resolution observations of a number of class I methanol maser transitions towards this source, including the $84-\mathrm{GHz}$ transition.
Voronkov et al. (2006) found that the 84-GHz methanol maser emission was spatially coincident with the $95-\mathrm{GHz}$ transition, with some spots shown to be coincident with a molecular outflow.

G 344.23-0.57. Voronkov et al. (2014) show that the 36- and $44-\mathrm{GHz}$ class I methanol maser emission is distributed right out to the full width at half-maximum (FWHM) of the ATCA beam at $7 \mathrm{~mm}$ (which is comparable to the Mopra beam), meaning that some of the $84-\mathrm{GHz}$ components may lie beyond the half-power points of the smaller $3 \mathrm{~mm}$ Mopra beam. The targeted position is close to the class II methanol maser location (Caswell et al. 2011) and we detect a narrow feature at the $38.3-\mathrm{GHz}$ methanol transition, but we detect no emission in either the 37.7- or the $38.5-\mathrm{GHz}$ transition. The velocity of the detected emission is at $-20.5 \mathrm{~km} \mathrm{~s}^{-1}$, identical to the peak velocity of the $36-\mathrm{GHz}$ transition. This velocity correspondence certainly gives credibility 


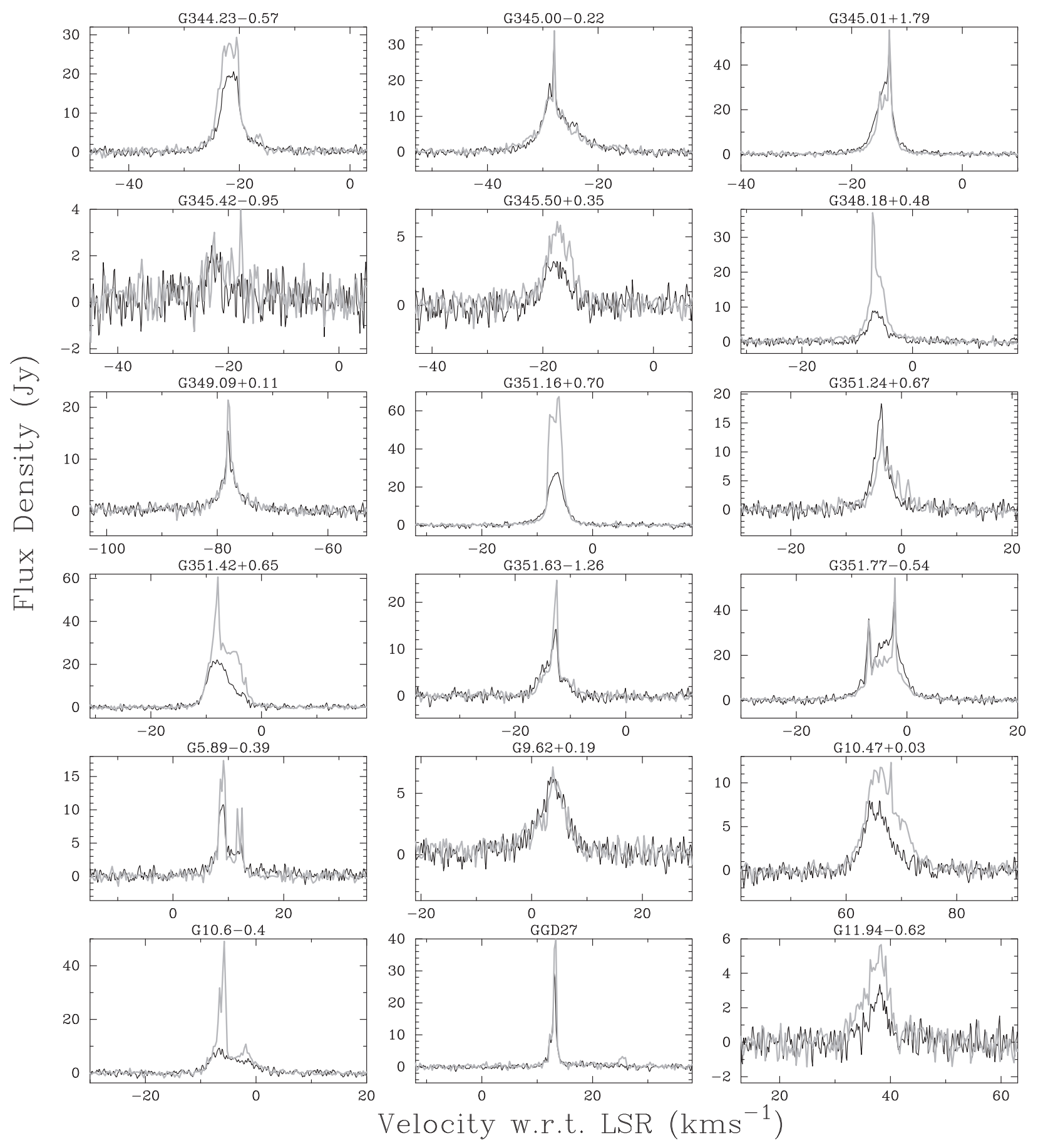

Figure 1. continued

to the narrow emission. Even though it is unusual to see emission in the $38.3-\mathrm{GHz}$ transition without corresponding 37.7- or 38.5$\mathrm{GHz}$ detections, there are examples of other sources where 38.3$\mathrm{GHz}$ emission is seen without accompanying $38.5-\mathrm{GHz}$ emission (e.g. G 335.79+0.17), and sources where the 37.7-GHz emission is significantly weaker than either the 38.3 - or the $38.5-\mathrm{GHz}$ transition (e.g. G 351.42+0.65).

We also detect emission from the 86.6- and 86.9-GHz transitions, which is likely to be thermal but shows some hints of narrow emission in the 86.6-GHz line. Methanol emission in the 88.9and $89.5-\mathrm{GHz}$ transitions is also detected.

G 345.01+1.79. Ellingsen et al. (2011) detected 37.7-, 38.3-, and 38.5-GHz emission towards this source, with peak flux densities of 207, 9.4, and 5.0 Jy, respectively. Their high-resolution observations showed some variation in the peak flux density in the time between the two sets of observations, with peak flux densities of 181, 9.3, and $5.9 \mathrm{Jy}$ for the respective transitions. We detected peak emission of 291,44 , and 21 Jy in our current observations, indicating significant 

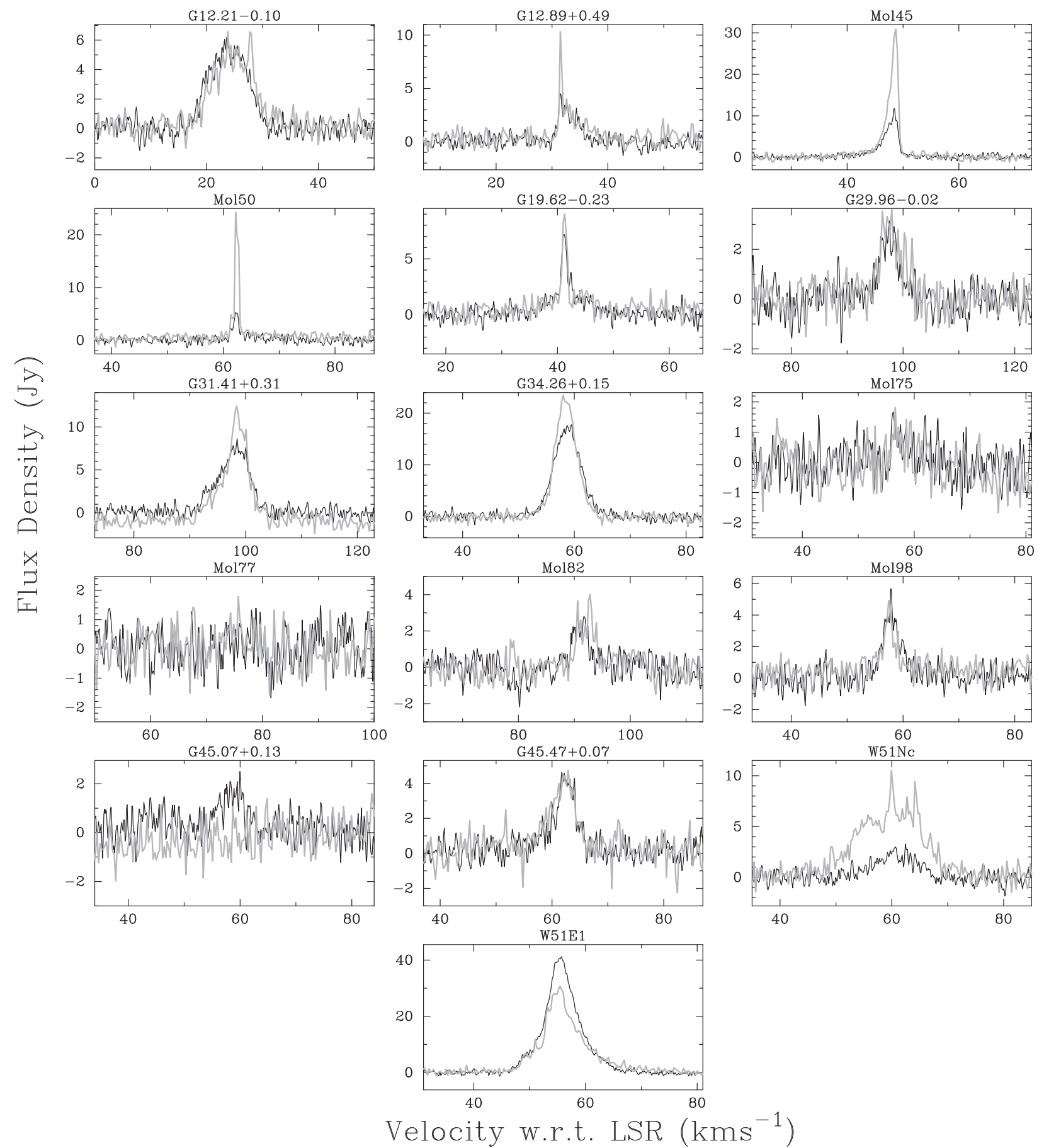

Figure 1. continued

variability between the 2011 observations of Ellingsen et al. (2011) and our 2018 observations.

This source also hosts one of the few known examples of 86.6- and 86.9-GHz maser emission, first detected by Cragg et al. (2001) with peak flux densities of 2.8 and $4.1 \mathrm{Jy}$ at a velocity of $\sim-21.7 \mathrm{~km} \mathrm{~s}^{-1}$. Further $86.6-\mathrm{GHz}$ observations by Ellingsen et al. (2003) revealed two spectral features with flux densities of 16.4 and $10 \mathrm{Jy}$ at velocities of -22.0 and $-21.2 \mathrm{~km} \mathrm{~s}^{-1}$. In our observations we find weak emission at $-21.2 \mathrm{~km} \mathrm{~s}^{-1}$ of about $1.5 \mathrm{Jy}$ and a main spectral feature at $-22.1 \mathrm{~km} \mathrm{~s}^{-1}$ of $\sim 8 \mathrm{Jy}$ at the two transitions.

$G 351.42+0.65$. This source, also known as NGC 6334F, has been observed at 37.3, 38.3, and $38.5 \mathrm{GHz}$ by Ellingsen et al. (2011) and Ellingsen et al. (2018) previously. Comparison of their peak flux densities with the current observations reveals some significant temporal variations in each of the 37.7-, 38.3-, and $38.5-\mathrm{GHz}$ 
Table 3. Characteristics of methanol masers detected at 37.7, 38.3, and $38.5 \mathrm{GHz}$. The first column gives the class I methanol maser source name and the next three groups of five columns give the minimum, maximum, and peak velocity, the peak flux density, and the integrated flux density for the 37.7-, 38.3-, and 38.5-GHz transitions, respectively. In the case where transitions are not detected, $3 \sigma$ detection limits are given. All new maser candidates are close to the $3 \sigma$ detection limit and have been marked with an ' $*$ ' following the peak flux density. These are discussed individually in Section 3.5 . References to previously detected sources are 1: Ellingsen et al. (2011); 2: Ellingsen et al. (2013); 3: Ellingsen et al. (2018); 4: Haschick et al. (1989).

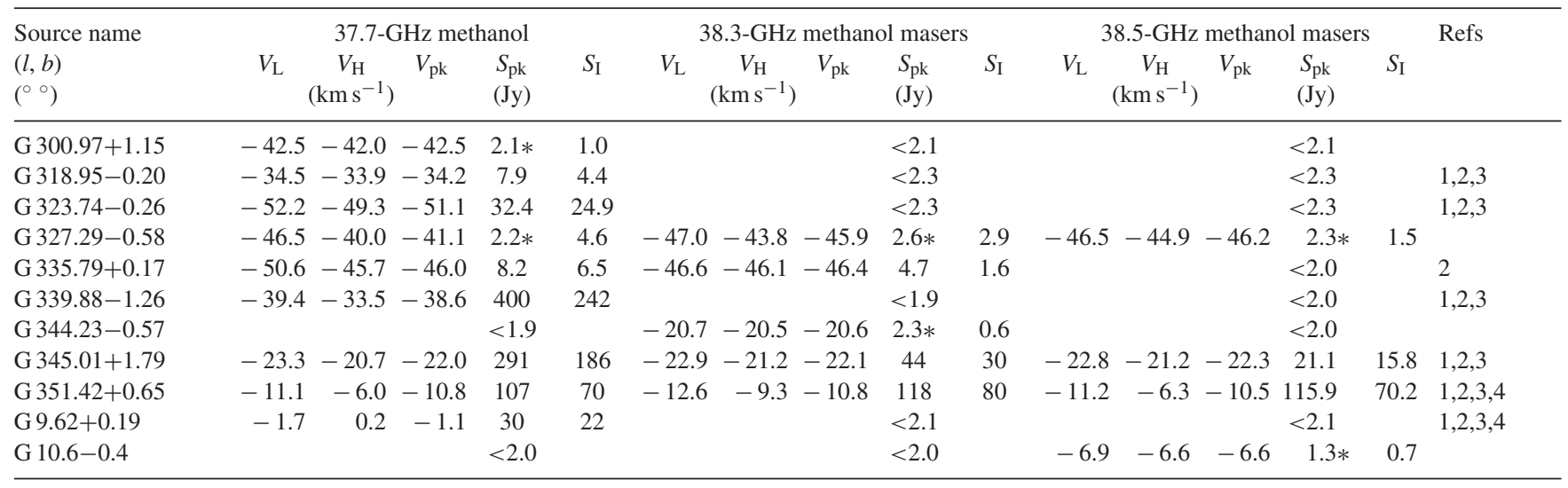

transitions [with flux densities of 70, 39, and 107 Jy for the 37.7GH transition; 174, 126, and 117 for the 38.3-GHz transition; and 150,151 , and 116 for the $38.5-\mathrm{GHz}$ transition in the Ellingsen et al. (2011), Ellingsen et al. (2018), and current observations, respectively].

$G 9.62+0.19$. This is the site of the highest peak flux density 6.7$\mathrm{GHz}$ methanol maser ever detected (e.g. Green et al. 2012, 2017). At 36 and $84 \mathrm{GHz}$ we detected almost identical emission in the two transitions, with peak flux densities of 3.8 and $3.4 \mathrm{Jy}$, respectively. At $37.7 \mathrm{GHz}$ we detected emission with a peak flux density of $30 \mathrm{Jy}$, slightly higher than previous observations (Ellingsen et al. 2011, 2013, 2018). Observations by Ellingsen et al. (2018) revealed weak emission in the $38.3-\mathrm{GHz}$ transition $(0.22 \mathrm{Jy})$, but no emission in the $38.5-\mathrm{GHz}$ transition.

G 10.6-0.4. We detect very weak emission in the $38.5-\mathrm{GHz}$ transition (peak flux density of $1.3 \mathrm{Jy}$ ) without detectable accompanying emission in either the 37.7- or the $38.3-\mathrm{GHz}$ transition. The weak $38.5-\mathrm{GHz}$ feature does share the same velocity as the peak feature in both the $36-\mathrm{GHz}$ emission and the $84-\mathrm{GHz}$ emission, lending some credibility to its authenticity. There are two nearby 6.7-GHz methanol maser sites (both of which may be associated with the W31 region: G 10.627-0.384 and G 10.629-0.333; Green et al. 2010) with velocity ranges that overlap with the $38.5-\mathrm{GHz}$ detection, so it is unclear where the emission is located. This site was observed by Ellingsen et al. (2011) but the emission was too weak (their rms is $1.1 \mathrm{Jy}$ at $38.4 \mathrm{GHz}$ ) to be detected. Further, more sensitive observations would be needed to confirm this detection.

$G$ 29.96-0.02. We detect narrow emission in both the 86.6- and 86.9-GHz methanol transitions, making it the fourth example of a maser in these transitions. The emission we detect shows slightly different peak velocities at the two transitions, but the $86.6-\mathrm{GHz}$ spectrum shows weak emission at the velocity of the $86.9-\mathrm{GHz}$ maser peak. The peak velocity of the associated 6.7-GHz methanol maser is at $96.0 \mathrm{~km} \mathrm{~s}^{-1}$ (Breen et al. 2015), slightly blueshifted compared to the 86.6- and 86.9-GHz detections, which at a velocity of $\sim 98 \mathrm{~km} \mathrm{~s}^{-1}$ still falls well within the overall $6.7-\mathrm{GHz}$ velocity range of 93.4 to $106.4 \mathrm{~km} \mathrm{~s}^{-1}$.

\section{DISCUSSION}

\subsection{Are the detected 36- and 84-GHz sources masers?}

Fig. 1 shows a number of different spectral profiles, ranging from narrow maser-like features (e.g. G 328.21-0.59) to broad thermallike components (e.g. G 327.39+0.20) and a combination of the two (e.g. G 331.13-0.24). From our single-dish observations alone, we cannot definitively confirm that all of the sources we detect are masers (or a combination of maser and thermal) since the implied lower limits on the brightness temperature do not exceed the expectations for kinetic temperatures in high-mass star formation regions, but we can make some arguments that they are likely to be based on previous observations. The bulk of the target list (71/94) has been taken from the Voronkov et al. (2014) sample of southern class I methanol masers at 36 and $44 \mathrm{GHz}$. These sources have all been scrutinized with interferometric observations and confirmed to host maser emission at both 36 and $44 \mathrm{GHz}$ (the rest of the sample is known to host maser emission at $44 \mathrm{GHz}$; Kurtz et al. 2004).

It is difficult to make a direct comparison of the $36-\mathrm{GHz}$ spectra presented in Fig. 1 with those in Voronkov et al. (2014) since the latter has high-resolution source maps that break the distributed maser emission into components while our spectra have blended all of the components into one single spectrum. However, comparison of some of the more 'thermal-looking' (i.e. broader and more Gaussian-like) sources in Fig. 1 with the corresponding spectra in Voronkov et al. (2014) shows that our single-dish observations have blended distinct maser components that are present in the higher-resolution data and that, in these sources, our flux densities are generally higher (although not by a consistent percentage). This suggests that the current single-dish spectra have additional thermal contributions that are resolved out in the interferometric observations.

Jordan et al. (2017) compared the spectral profiles of 44$\mathrm{GHz}$ class II methanol masers derived from the auto- and crosscorrelation data from the same interferometric observations taken with the Australia Telescope Compact Array. They found that, for their 77 maser sites, there was a huge distribution in the difference between the flux density of the two spectra, ranging from very 


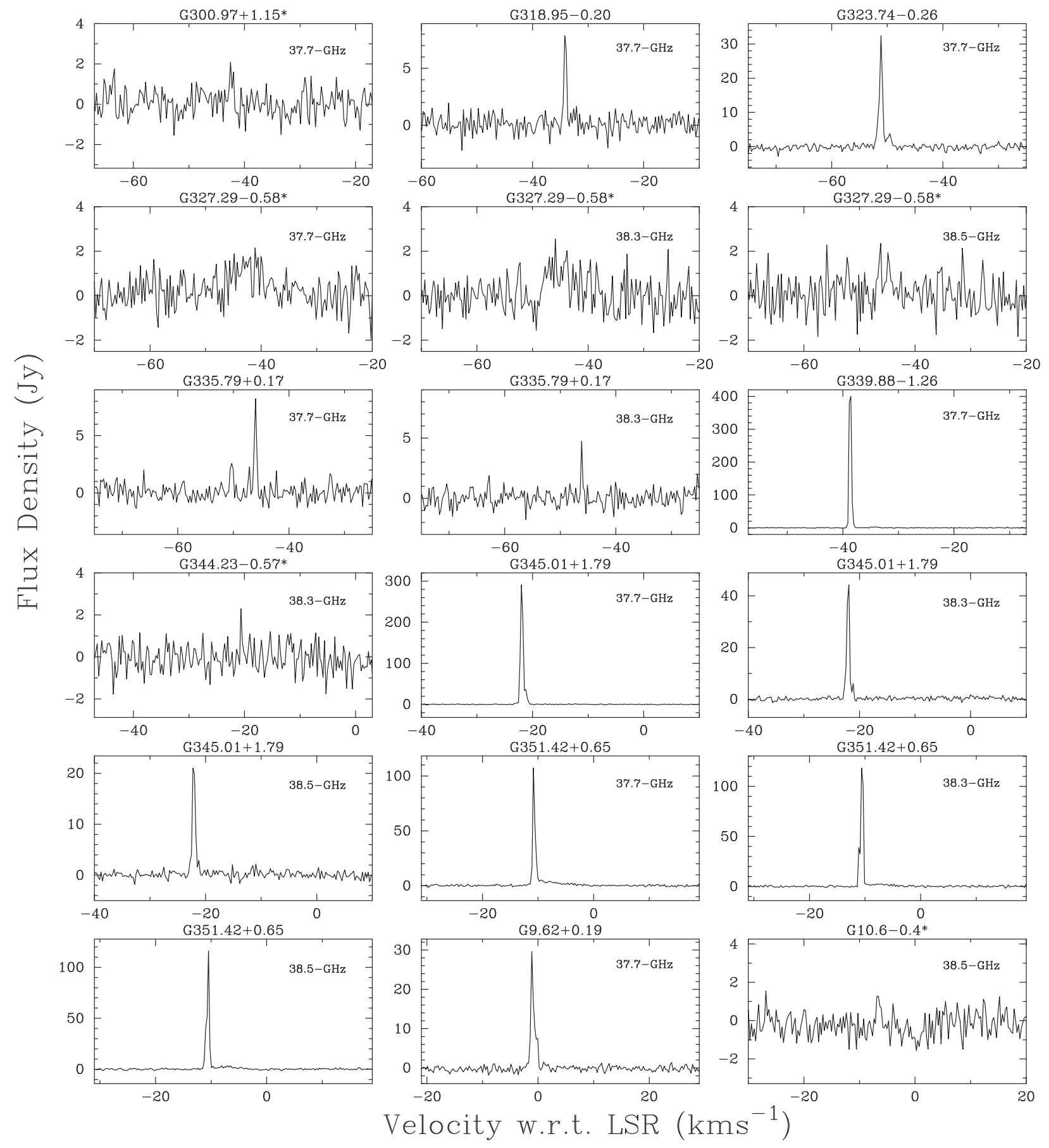

Figure 2. Spectra of the 37.7-, 38.3-, and 38.5-GHz sources detected towards class I methanol maser sources. New candidate detections are marked with an ' $*$ ' following the source name.

similar to sources where about $\sim 70$ per cent of the flux density was resolved out in the cross-correlation data (they had baselines up to $1.5 \mathrm{~km} \mathrm{~s}^{-1}$ ).

Since it is clear that all of the $36-\mathrm{GHz}$ sources in Fig. 1 that have been observed with interferometry (the majority) host maser emission, even those that have spectral profiles reminiscent of thermal emission in the current observations, it follows that the very similar 84-GHz spectra also contain maser emission, even in the case that they too appear to have thermal-like spectral profiles. However, comparing the $36-\mathrm{GHz}$ flux densities of our current observations with those of Voronkov et al. (2014), combined with the findings of Jordan et al. (2017) for 44-GHz class I methanol masers, it is also likely that in a number of cases our single-dish spectra also have contributions from thermal emission.

\subsection{Previous 84-GHz observations: detection rates and nature of the emission}

Previous targeted class I methanol maser searches by Kalenskii et al. (2001) and Rodríguez-Garza et al. (2018) (both with single- 


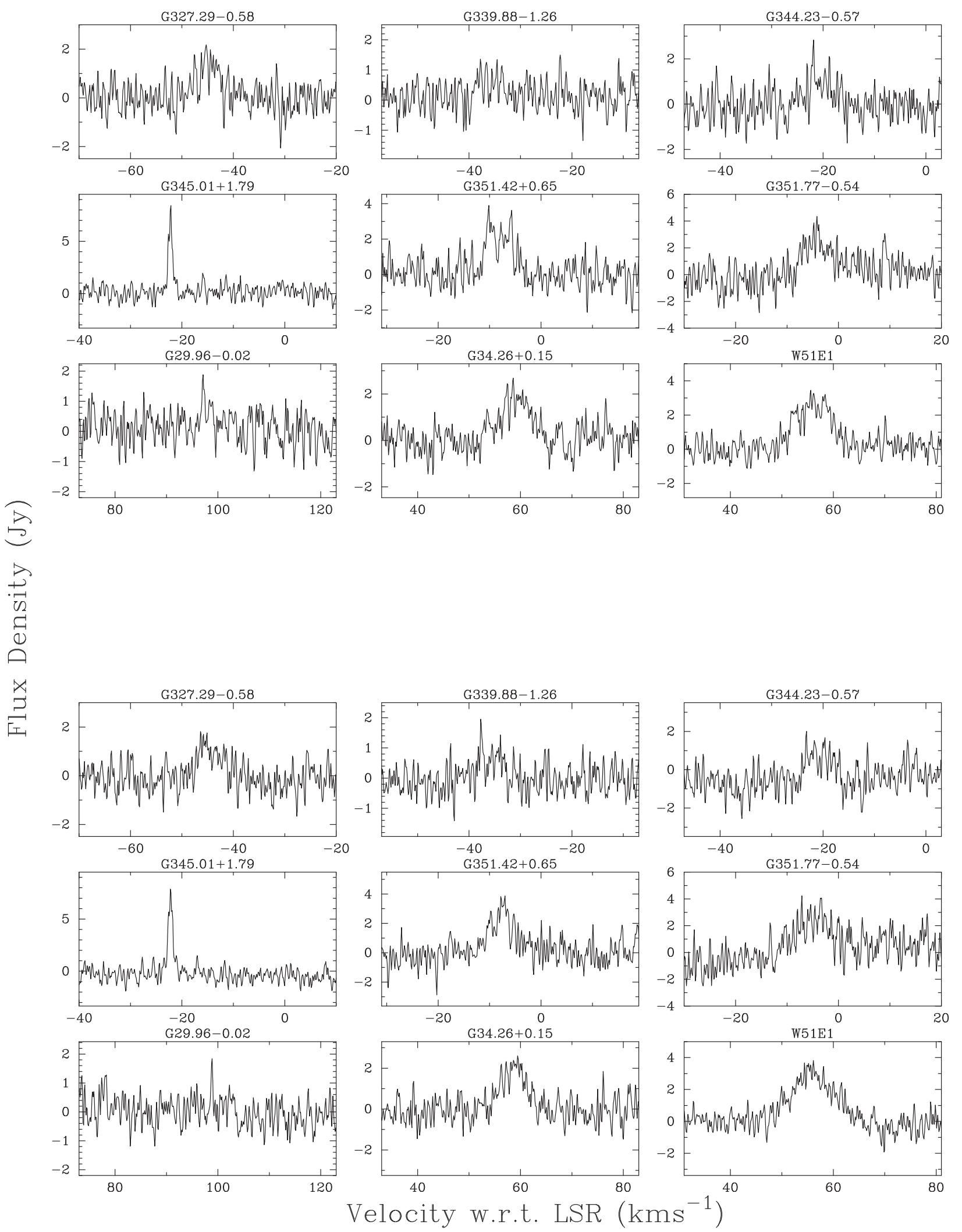

Figure 3. Spectra of the $86.6-\mathrm{GHz}$ (top) and $86.9-\mathrm{GHz}$ (bottom) sources detected towards class I methanol masers. 
Table 4. Detections of methanol emission at 86.6 and $86.9 \mathrm{GHz}$. The first column gives the class I methanol maser source name and the next two groups of five columns give the minimum, maximum, and peak velocity, the peak flux density, and integrated flux density for the 86.6- and 86.9-GHz transitions, respectively. References to previously detected sources are 1: Ellingsen et al. (2003); 2: Cragg et al. (2001); 3: Minier \& Booth (2002).

\begin{tabular}{|c|c|c|c|c|c|c|c|c|c|c|c|}
\hline \multirow{2}{*}{$\begin{array}{l}\text { Source name } \\
(l, b) \\
\left({ }^{\circ} \circ\right)\end{array}$} & \multicolumn{5}{|c|}{ 86.6-GHz methanol masers } & \multicolumn{5}{|c|}{ 86.9-GHz methanol masers } & \multirow[t]{2}{*}{ Refs } \\
\hline & $V_{\mathrm{L}}$ & $\begin{array}{c}V_{\mathrm{H}} \\
\left(\mathrm{km} \mathrm{s}^{-1}\right)\end{array}$ & $V_{\mathrm{pk}}$ & $\begin{array}{l}S_{\mathrm{pk}} \\
(\mathrm{Jy})\end{array}$ & $\begin{array}{c}S_{\mathrm{I}} \\
\left(\mathrm{Jy} \mathrm{km} \mathrm{s}^{-1}\right)\end{array}$ & $V_{\mathrm{L}}$ & $\begin{array}{c}V_{\mathrm{H}} \\
\left(\mathrm{km} \mathrm{s}^{-1}\right)\end{array}$ & $V_{\mathrm{pk}}$ & $\begin{array}{l}S_{\mathrm{pk}} \\
(\mathrm{Jy})\end{array}$ & $\begin{array}{c}S_{\mathrm{I}} \\
\left(\mathrm{Jy} \mathrm{km} \mathrm{s}^{-1}\right)\end{array}$ & \\
\hline G $339.88-1.26$ & -38.4 & -33.6 & -38.2 & 1.4 & 1.5 & -38.2 & -33.9 & -37.7 & 2.0 & 1.4 & \\
\hline G 344.23-0.57 & -25.1 & -16.4 & -21.8 & 2.8 & 4.2 & -23.3 & -16.8 & -23.2 & 2.0 & 2.7 & \\
\hline G $345.01+1.79$ & -22.8 & -15.6 & -22.1 & 8.4 & 8.3 & -23.5 & -17.0 & -22.2 & 7.9 & 8.3 & 1,2 \\
\hline G $351.42+0.65$ & -11.3 & -4.0 & -10.1 & 3.9 & 13.8 & -10.6 & -3.9 & -7.0 & 3.9 & 13.6 & 1,2 \\
\hline G 29.96-0.02 & 97.0 & 98.4 & 97.1 & 1.9 & 1.0 & 98.8 & 99.0 & 98.9 & 1.8 & 0.5 & \\
\hline G $34.26+0.15$ & 57.0 & 61.4 & 58.6 & 2.7 & 6.7 & 56.1 & 61.2 & 59.5 & 2.6 & 7.8 & \\
\hline W51E1 & 49.7 & 61.4 & 55.6 & 3.4 & 19.0 & 49.7 & 62.3 & 56.1 & 3.8 & 24.4 & 3 \\
\hline
\end{tabular}

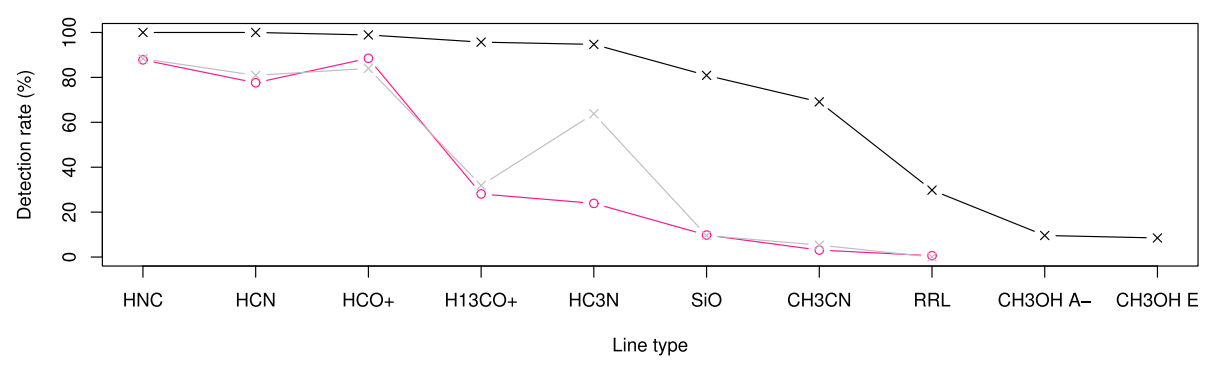

Figure 4. Detection rates (back crosses; corresponding to 100, 100, 98.9, 95.7, 94.7, 80.9, 69.1, 29.8, 9.6, 8.5 per cent) of the molecular and radio recombination lines observed. The detection rates of the MALT90 survey of 3246 dense clumps across the Galaxy are shown by pink open circles for comparison (Rathborne et al. 2016). The MALT90 observations did not include the 88.9- $\left(\mathrm{CH}_{3} \mathrm{OH} A^{-}\right)$and $89.5-\mathrm{GHz}\left(\mathrm{CH}_{3} \mathrm{OH} E\right)$ thermal methanol transitions, so we cannot compare the detection rates for those two lines. The grey line shows what our detection rates would be at the MALT90 95 per cent completeness level $\left(T_{A}^{*}>0.4 \mathrm{~K}\right.$; Rathborne et al. 2016).

dish telescopes) have resulted in 84-GHz detection rates of 94 and 74 per cent, respectively. The latter target list was exclusively made up of 44-GHz targets, and the former is likely to be, although the authors just state that they are class I maser targets. Kalenskii et al. (2001) reported that the majority of their sources (34/48) were likely to be quasi-thermal rather than simply maser emission based on their broad linewidths, especially when compared to their $44-\mathrm{GHz}$ methanol maser counterparts. The spectral resolution $\left(100 \mathrm{~km} \mathrm{~s}^{-1}\right)$ accounts for the slightly lower detection rate in Rodríguez-Garza et al. (2018) and prohibits them from assessing spectral profiles or intensities.

Unlike Kalenskii et al. (2001), who compared their $84-\mathrm{GHz}$ spectra to the $44-\mathrm{GHz}\left(7_{0} \rightarrow 6_{1} A^{+}\right)$transition, we find the spectral profiles for our two transitions to be remarkably similar. The 44$\mathrm{GHz}$ transition is not in the same transition family as the 36- and 84-GHz transitions, so it is much less likely to have a similar spectral profile. Interferometric observations of $84-\mathrm{GHz}$ methanol sources are very limited, but the one source in our sample that has been observed at high spatial resolution (Voronkov et al. 2006) was found to harbour maser emission. For these, and the reasons outlined in Section 4.1, we believe our sources contain maser emission; however, further high spatial resolution observations would be required to definitely understand the nature of the detected $84-\mathrm{GHz}$ emission.

\subsection{Comparison of the 36- and 84-GHz spectral profiles}

Spectra of both the 36- and 84-GHz methanol maser lines are presented in Fig. 1 for each target, showing remarkably similar spectral profiles in the majority of cases. These $36-$ and $84-\mathrm{GHz}$ methanol lines are the result of consecutive transitions of the same ladder, the next of which (the $6_{-1}-5_{0} E$ transition at $133 \mathrm{GHz}$ ) has also been found to closely resemble the spectral profiles of $84-\mathrm{GHz}$ emission (Kalenskii et al. 2001). While it is somewhat expected for pairs of transitions to show similar spectral profiles, our own observations show that the 36- and $84-\mathrm{GHz}$ pair are much more alike than other cases such as the 86.6- and 86.9-GHz transitions, which are the next in sequence in the series that produces the 38.3- and 38.5-GHz lines. In another example, McCarthy et al. (2018a) used their interferometric data to compare the positions and velocities of both $44\left(7_{0} \rightarrow 6_{1} A^{+}\right)$and $95\left(8_{0} \rightarrow 7_{1} A^{+}\right) \mathrm{GHz}$ methanol maser features. They found that 49 per cent of $95-\mathrm{GHz}$ maser components had accompanying 44-GHz emission whereas we find few examples of $84-\mathrm{GHz}$ emission devoid of a $36-\mathrm{GHz}$ counterpart (although higher spatial resolution would be needed to confirm this). Kim, Kim \& Park (2018) conducted simultaneous 44- and 95-GHz observations, detecting 44-GHz emission towards 83 sources and accompanying $95-\mathrm{GHz}$ methanol maser emission towards 68 of those, also indicating a slightly less close relationship than for the 36- and 84-GHz transitions.

Fig. 5 shows a comparison of the velocity of the peak $36-\mathrm{GHz}$ and 84-GHz methanol emission, revealing a tight correlation between the peak velocities of the 92 sources detected at both frequencies. Only eight sources show 36 - and $84-\mathrm{GHz}$ peak velocity differences of more than $2 \mathrm{~km} \mathrm{~s}^{-1}$; 75 show velocities within $1 \mathrm{~km} \mathrm{~s}^{-1}$ of each other and 67 of those are within $0.5 \mathrm{~km}^{-1}$. The largest peak velocity difference is $5.2 \mathrm{~km} \mathrm{~s}^{-1}$ in G 345.42-0.95, which is a rare example of a source that shows no $84-\mathrm{GHz}$ emission at the $36-\mathrm{GHz}$ 


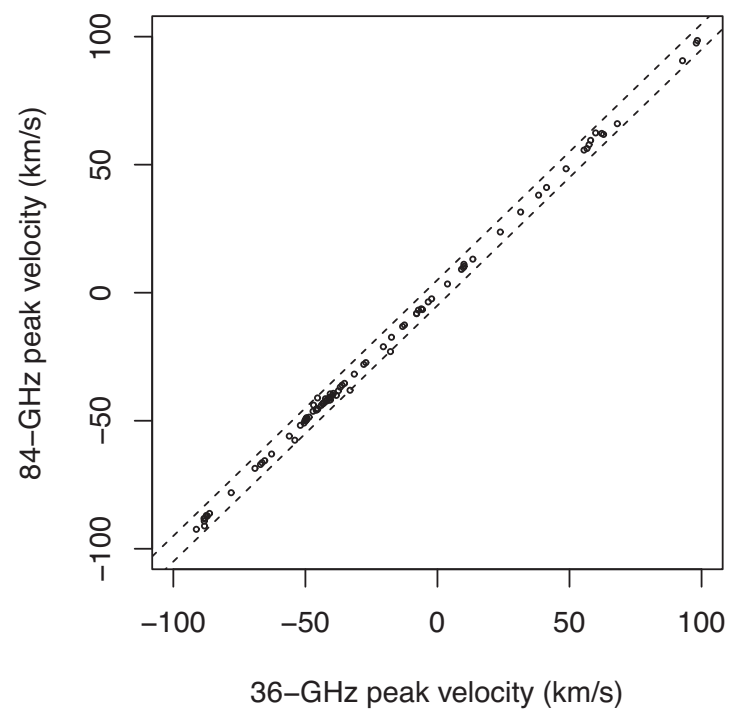

Figure 5. Peak velocity of the $36-\mathrm{GHz}$ compared to $84-\mathrm{GHz}$ methanol masers. The two dashed lines show a deviation of $5 \mathrm{~km} \mathrm{~s}^{-1}$ either side of unity.

peak. Inspection of the Voronkov et al. (2014) high spatial resolution data shows that the $36-\mathrm{GHz}$ peak emission that we detected at $-17.8 \mathrm{~km} \mathrm{~s}^{-1}$ lies well beyond the HPBW of the $3 \mathrm{~mm}$ Mopra beam, so further observations would be needed to rule out $84-\mathrm{GHz}$ emission at that location.

The mean and median velocity ranges are slightly higher for the $36-\mathrm{GHz}$ sources compared to the $84-\mathrm{GHz}$ sources: $10.1 \pm 0.8$ and 9.2 compared with $7.7 \pm 0.5$ and $7.1 \mathrm{~km} \mathrm{~s}^{-1}$, respectively. The 36-GHz velocity ranges fall between 0.2 and $48.3 \mathrm{~km} \mathrm{~s}^{-1}$ and the $84-\mathrm{GHz}$ sources between 0.6 and $34.8 \mathrm{~km} \mathrm{~s}^{-1}$. The sources with the largest velocity ranges are G $341.19-0.23$ at $36 \mathrm{GHz}$ and $\mathrm{G} 328.81+0.63$ at $84 \mathrm{GHz}$.

Fig. 6 shows box plots of the peak velocity of $\mathrm{HC}_{3} \mathrm{~N}, \mathrm{HNC}$, $\mathrm{HCO}^{+}, \mathrm{HCN}, \mathrm{SiO}$, and $\mathrm{H}^{13} \mathrm{CO}^{+}$with respect to the peak velocity of the associated 36- and 84-GHz emission. In all cases the median velocity difference is close to zero, but it is clear that some molecules show better velocity agreement with the 36- and 84$\mathrm{GHz}$ methanol masers than others. In most instances this is due to significant self-absorption in some of the lines with high optical depths, such as HCN, which is more often blueshifted with respect to the maser velocity, consistent with the detection of significant emission on the nearside of the source. From these comparisons it appears that the best maser velocity correspondence is with $\mathrm{HNC}, \mathrm{HC}_{3} \mathrm{~N}$, and $\mathrm{H}^{13} \mathrm{CO}^{+}$(the latter two are likely to be optically thin).

In an analysis of 44-GHz class I methanol masers, Jordan et al. (2017) found that class I methanol masers were better indicators of systemic velocities than class II methanol masers. Comparing the peak 44-GHz methanol maser velocity to CS (1-0), they found a mean velocity difference of $0.09 \pm 0.18 \mathrm{~km} \mathrm{~s}^{-1}$, a median velocity difference of $0.04 \mathrm{~km} \mathrm{~s}^{-1}$, and a standard deviation of $1.56 \mathrm{~km} \mathrm{~s}^{-1}$. Table 5 shows the mean, median, and standard deviations of the $\mathrm{HC}_{3} \mathrm{~N}, \mathrm{HNC}$, and $\mathrm{H}^{13} \mathrm{CO}^{+}$peak velocities with respect to the $36-$ and $84-\mathrm{GHz}$ methanol maser peak velocities, showing that they are comparable to those found by Jordan et al. (2017) when comparing the $44-\mathrm{GHz}$ peak velocities to that of CS (1-0).

\subsection{Comparison between the 36- and 84-GHz flux densities}

The 92 36-GHz methanol maser detections range in peak flux density from 1.8 to $245 \mathrm{Jy}$ (mean of 28.1 , median $14.3 \mathrm{Jy}$ ) and the $9384-\mathrm{GHz}$ detections range in peak flux density from 1.2 to $68 \mathrm{Jy}$ (mean of 13.0 and median of $8.7 \mathrm{Jy}$ ). The strongest $36-\mathrm{GHz}$ maser is G 335.59-0.29 and the strongest 84-GHz source is G 327.29-0.58. A comparison between the 36- and 84-GHz peak and integrated flux densities is shown in Fig. 7. The correlation coefficient between the peak and integrated flux densities of the two transitions are 0.52 and 0.71 , indicating moderate and strong positive correlations. The fact that the integrated flux densities are more tightly correlated than the respective peak flux densities is reflected in Fig. 7 and indicates that the integrated flux densities are more robust to more extreme differences that might be seen in only a single velocity feature. The mean and median 36- to $84-\mathrm{GHz}$ peak flux density ratio are 2.4 and 1.6, respectively. The $2736-\mathrm{GHz}$ methanol masers with peak flux densities that surpass $30 \mathrm{Jy}$ have relatively higher $36-$ to $84-\mathrm{GHz}$ peak flux density ratios with a mean value of 4.1 and a median of 2.4 .

The average $84-\mathrm{GHz}$ integrated flux density is $46.8 \mathrm{Jy} \mathrm{km} \mathrm{s}^{-1}$ and the median is $29.4 \mathrm{Jy} \mathrm{km} \mathrm{s}^{-1}$ compared with the $36-\mathrm{GHz}$ sample, which has an average of $77.0 \mathrm{~km} \mathrm{~s}^{-1} \mathrm{Jy}$ and median of $41.3 \mathrm{Jy} \mathrm{km} \mathrm{s}^{-1}$. The average $36-$ to $84-\mathrm{GHz}$ integrated flux density ratio is 2.6 and the median is 1.4 . The ratio of these lines is similar to that of the $84-$ to $133-\mathrm{GHz}$ transitions, reported by Kalenskii et al. (2001) to be 1.4. Fig. 8 shows the distribution of integrated flux density ratios for the full sample, along with the ratio for both $36-$ and $84-\mathrm{GHz}$ masers that have integrated flux densities of more than $50 \mathrm{Jy} \mathrm{km} \mathrm{s}^{-1}$. For $36-\mathrm{GHz}$ masers with integrated flux densities greater than $50 \mathrm{Jy} \mathrm{km} \mathrm{s}^{-1}$, the mean and median ratio is 3.8 and 2.1 , compared to 1.9 and 1.5 for the $84-\mathrm{GHz}$ that have integrated flux densities greater than $50 \mathrm{Jy} \mathrm{km} \mathrm{s}^{-1}$.

There are 16 cases where the $84-\mathrm{GHz}$ peak flux density surpasses that of the $36-\mathrm{GHz}$ methanol maser counterpart, 15 of which also have higher integrated intensities. There are an additional eight sources that have a larger $36-\mathrm{GHz}$ peak flux density but have a higher $84-\mathrm{GHz}$ integrated flux density. The source with the largest $84-$ to $36-\mathrm{GHz}$ integrated flux density ratio is G 301.14-0.2.

Although the 36- and 84-GHz methanol maser transitions have similar optimal conditions (e.g. Leurini et al. 2016), there are conditions that favour the 36- or 84-GHz transitions differently. For example, at high densities $\left(10^{6}-10^{8} \mathrm{~cm}^{-3}\right), 36-$ to $84-\mathrm{GHz}$ ratios close to 1 might be expected but lower densities $\left(10^{3}-10^{6} \mathrm{~cm}^{-3}\right)$ can favour the $36-\mathrm{GHz}$ transition, resulting in $36-$ to $84-\mathrm{GHz}$ ratios of $\sim 2$ (McEwen et al. 2014).

Fig. 7 also highlights the targets where radio recombination lines have also been detected. In previous studies, it has been suggested that other types of masers show a change in luminosity with evolution (e.g. Breen et al. 2010) and if we consider the presence of a detectable recombination line as an indication of a slightly more evolved site, Fig. 7 shows that there is no simple trend whereby the line ratio of the 36 - to $84-\mathrm{GHz}$ sources changes with evolution. Given that class I methanol masers trace shocks, they can be associated with multiple phases in the evolution of a young high-mass star (such as outflows and expanding $\mathrm{H}$ II regions; Voronkov et al. 2014), and it is not surprising that there is no simple evolutionary trend. Further complicating the issue is the large Mopra beam which may lead to confusion between the multiple detected lines. 

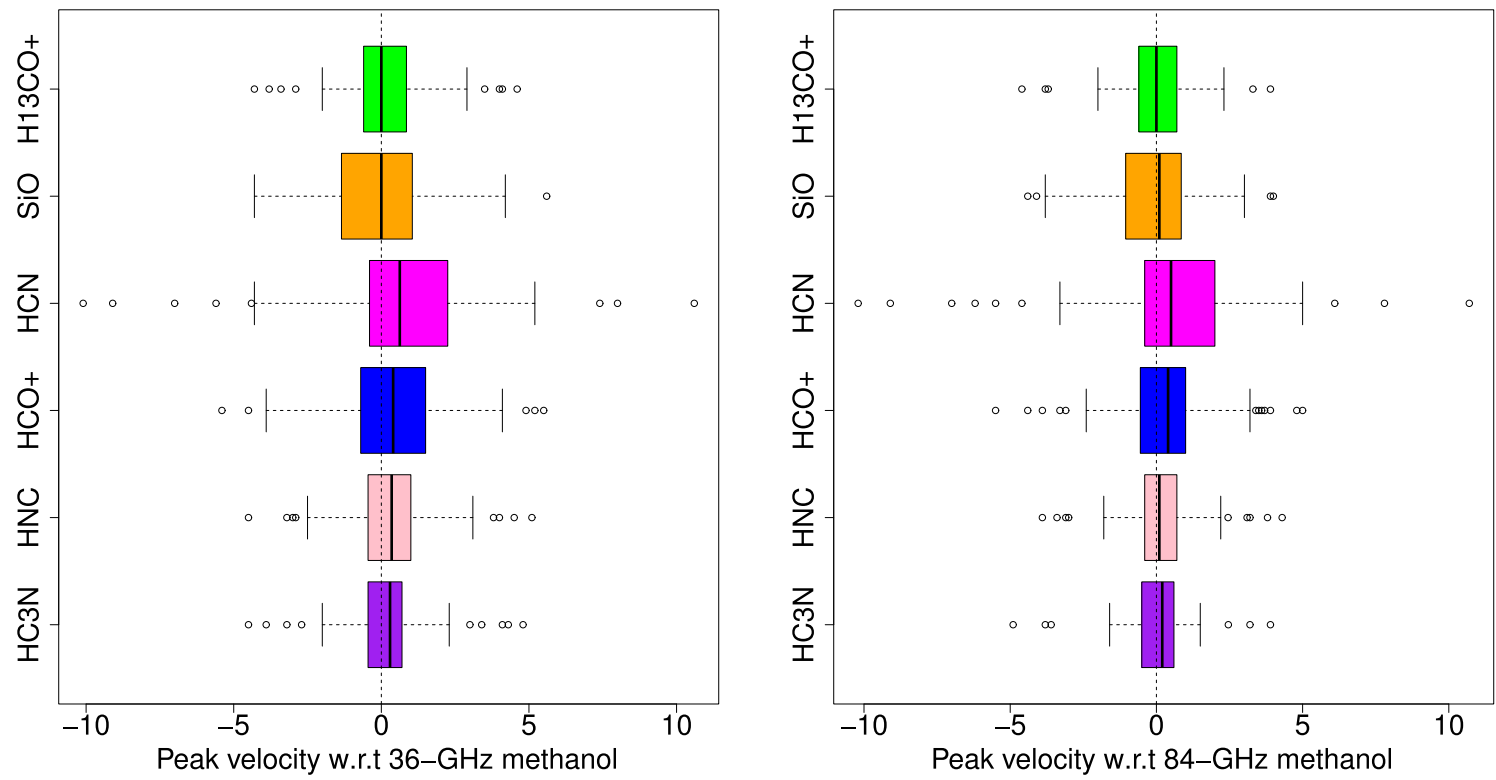

Figure 6. Box plots of the peak velocity of $\mathrm{HC}_{3} \mathrm{~N}, \mathrm{HNC}, \mathrm{HCO}^{+}, \mathrm{HCN}, \mathrm{SiO}$, and $\mathrm{H}^{13} \mathrm{CO}^{+}$with respect to the peak velocity of the $36-\mathrm{GHz}$ (left) and $84-\mathrm{GHz}$ (right) emission. The vertical dashed line shows equal velocities. In each box plot, the solid vertical black line represents the median of the data, the coloured box represents the interquartile range (25th to 75 th percentile), and the dashed horizontal lines (the 'whiskers') show the range from the 25 th percentile to the minimum value and the 75 th percentile to the maximum value, respectively. Values that fall more than 1.5 times the interquartile range from either the 25 th or the 75th percentile are considered to be outliers and are represented by open circles.

Table 5. Mean (with standard error), median, and standard deviations of the velocity of molecular lines with respect to the 36- and 84-GHz methanol maser velocities in units of $\mathrm{km} \mathrm{s}^{-1}$.

\begin{tabular}{|c|c|c|c|c|c|c|}
\hline \multirow[t]{2}{*}{ Line } & \multicolumn{3}{|c|}{ 36-GHz masers } & \multicolumn{3}{|c|}{ 84-GHz masers } \\
\hline & Mean & Median & s.d. & Mean & Median & s.d. \\
\hline $\mathrm{HC}_{3} \mathrm{~N}$ & $0.22 \pm 0.16$ & 0.29 & 1.46 & $0.05 \pm 0.12$ & 0.2 & 1.17 \\
\hline $\mathrm{HNC}$ & $0.30 \pm 0.16$ & 0.35 & 1.54 & $0.13 \pm 0.14$ & 0.1 & 1.32 \\
\hline $\mathrm{H}^{13} \mathrm{CO}^{+}$ & $0.10 \pm 0.16$ & 0 & 1.51 & $-0.04 \pm 0.13$ & 0 & 1.24 \\
\hline
\end{tabular}
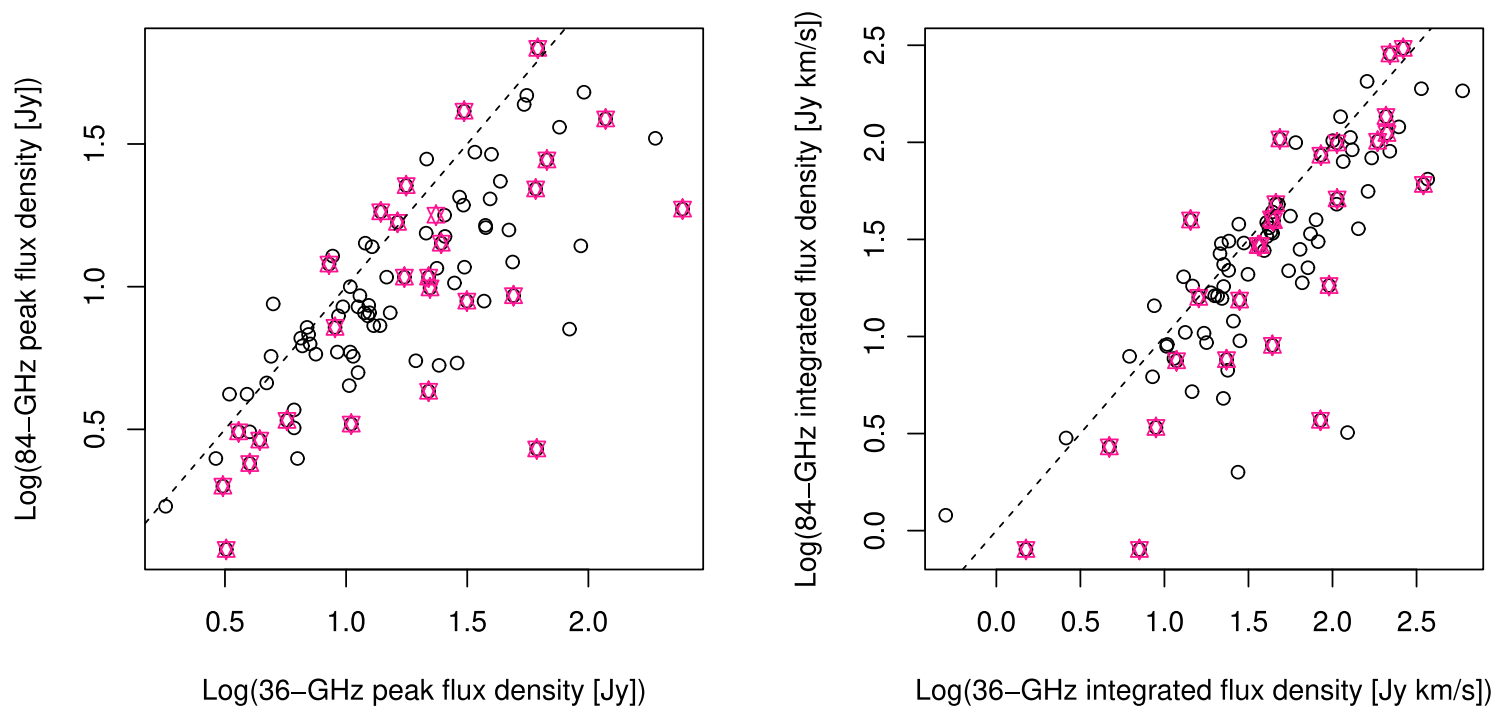

Figure 7. Log-log plots of the peak (left) and integrated (right) 36- versus $84-\mathrm{GHz}$ methanol maser flux density. The dashed lines show $x=y$. Pink stars distinguish those sources that also exhibit radio recombination lines (28/92). 


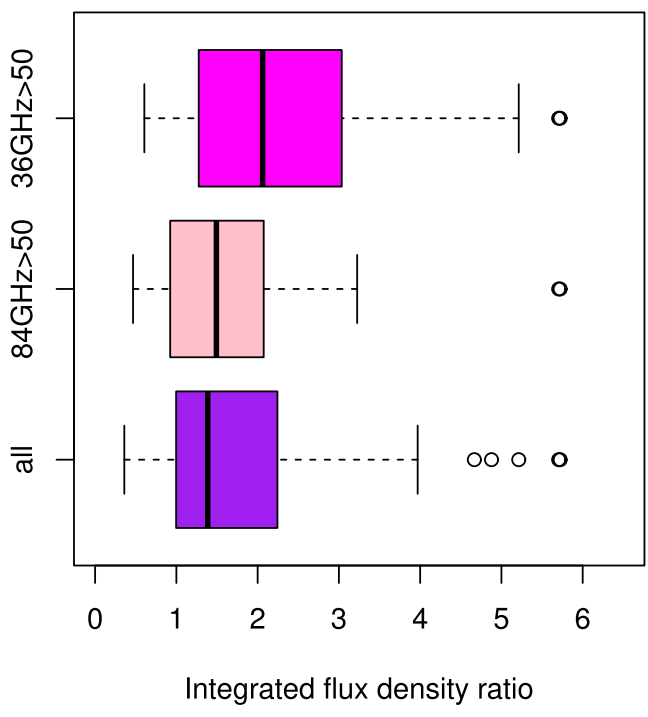

Figure 8. Box plots showing the ratio of $36-$ to $84-\mathrm{GHz}$ integrated flux densities for the full sample of 92 sources with detections at both frequencies (purple), the 24 sources with $84-\mathrm{GHz}$ integrated flux densities greater than $50 \mathrm{Jy} \mathrm{km} \mathrm{s}^{-1}$ (pink), and the 36 sources with 36-GHz integrated flux densities greater than $50 \mathrm{Jy} \mathrm{km} \mathrm{s}^{-1}$ (magenta). Note that there are four sources with ratios greater than $6(8.9,13.7,22.9$, and 38.4), the two highest of which have $36-\mathrm{GHz}$ integrated flux densities greater than $50 \mathrm{Jy} \mathrm{km} \mathrm{s}^{-1}$. See Fig. 6 for a general explanation of box plots.

\subsection{Comparison of the class I methanol masers with thermal molecular lines}

Fig. 4 shows the detection rates of the thermal molecular and recombination lines we detect (excluding $\mathrm{H}^{13} \mathrm{CN}$, since the frequency coverage excludes the velocities of most of the targets), highlighting the high association rates of most of the observed lines with our maser-associated star formation regions. The fact that $\mathrm{HNC}, \mathrm{HCN}$, and $\mathrm{HCO}^{+}$were all detected towards 93 of the 94 target sources very strongly indicates the presence of dense gas at the locations of all of the target class I methanol masers. In the one case where $\mathrm{HCO}^{+}$is not reported in Table A1 (towards G 335.06-0.43), $\mathrm{H}^{13} \mathrm{CO}^{+}$was, and there are hints of some narrow emission at the right velocity of the $\mathrm{HCO}^{+}$spectrum, indicating that it is significantly self-absorbed.

Also present in Fig. 4 are the MALT90 detection rates of eight of our lines towards a sample of 3246 high-mass clumps (Rathborne et al. 2016). While MALT90 has similarly high detection rates of $\mathrm{HNC}, \mathrm{HCN}$, and $\mathrm{HCO}^{+}$, there is a significant drop off in the detection rates of $\mathrm{H}^{13} \mathrm{CO}^{+}, \mathrm{HC}_{3} \mathrm{~N}, \mathrm{SiO}, \mathrm{CH}_{3} \mathrm{CN}$, and radio recombination line emission. This is mostly explained by the higher sensitivity of the current observations, as can be seen by the excellent agreement between the MALT90 detection rates and our detection rates adjusted to the MALT90 95 per cent completeness level, shown by the grey crosses in Fig. 4 ( $T_{A}^{*}>0.4 \mathrm{~K}$; Rathborne et al. 2016). The notable exception is $\mathrm{HC}_{3} \mathrm{~N}$, which has much higher detection rates towards the class I methanol maser selected sample. We have compared MALT90 detection rates as a function of source temperatures (from Guzmán et al. 2015) and also their MALT90defined evolutionary categories, which indicate that, in order to account for the high $\mathrm{HC}_{3} \mathrm{~N}$ detection rates, the class I methanol maser targets are (in general) likely to be both protostellar and warm.

Fig. 9 shows the 36- and $84-\mathrm{GHz}$ methanol maser integrated flux density plotted against the integrated $\mathrm{HC}_{3} \mathrm{~N}, \mathrm{HNC}, \mathrm{HCO}^{+}, \mathrm{HNC}$, $\mathrm{SiO}$, and $\mathrm{H}^{13} \mathrm{CO}^{+}$intensities. The correlation coefficients mostly indicate moderately correlated positive relationships, suggesting that the 36- and $84-\mathrm{GHz}$ sources with higher integrated flux densities are generally associated with molecular lines with higher integrated intensities. The slopes of the fitted linear relationship in each case are similar $(0.41 \pm 0.06,0.31 \pm 0.06,0.31 \pm 0.06$, $0.33 \pm 0.07,0.56 \pm 0.07$, and $0.29 \pm 0.05$ for the $36-\mathrm{GHz}$ maser, and $0.51 \pm 0.05,0.38 \pm 0.05,0.37 \pm 0.07,0.42 \pm 0.06,0.60, \pm 0.07$, and $0.36 \pm 0.04$ for the $84-\mathrm{GHz}$ maser line), indicating that the integrated flux density of the maser lines scales with the overall quantity of gas. For an optically thin gas, the intensity scales linearly with the abundance of a given molecule; however, for the same temperature it has an exponential dependence on the excitation energy. This, together with inadequate sensitivity, could account for the very low detection rate of the 88.9- $\left(\mathrm{CH}_{3} \mathrm{OH} A^{-}\right)$and 89.5- $\mathrm{GHz}\left(\mathrm{CH}_{3} \mathrm{OH} E\right)$ thermal methanol transitions. Furthermore, the spatial resolution of the current observations is insufficient to resolve the regions of gas where the masers originate, so determining the exact relationship would require higher resolution follow-up observations.

Comparison of the 44-GHz class I methanol integrated flux densities (derived from their autocorrelated data) with the integrated intensities of $\mathrm{CS}(1-0), \mathrm{SiO}(1-0)$, and $\mathrm{CH}_{3} \mathrm{OH} 1_{0}-0_{0} A^{+}$by Jordan et al. (2017) similarly found moderately correlated positive relationships (correlation coefficients of 0.41, 0.57, and 0.40, respectively) between the masers and the thermal line emission. They suggested that this could indicate that the more luminous 44$\mathrm{GHz}$ methanol masers may be associated with the more massive high-mass star formation regions. Interestingly, Jordan et al. (2017) find that the integrated intensity of the 44-GHz masers had a closer relationship with the integrated intensity of the $\mathrm{SiO}(1-0)$ emission than the other lines. In our targeted observations we find that the tightest linear relationships in Fig. 9 are with $\mathrm{HC}_{3} \mathrm{~N}$ and $\mathrm{SiO}(2-1)$, which have Pearson correlation coefficients with the integrated 36$\mathrm{GHz}$ methanol masers of 0.61 and 0.69 , and with the integrated $84-\mathrm{GHz}$ methanol masers of 0.74 and 0.73 . This indicates that there is an even closer relationship between the $\mathrm{SiO}(2-1)$ and the 36- and $84-\mathrm{GHz}$ integrated intensities than that of the $44-\mathrm{GHz}$ methanol masers and $\mathrm{SiO}(1-0)$. The close intensity correlation between the collisionally excited class I methanol masers and $\mathrm{SiO}$ probably is a reflection of the fact that they are both tracers of shocked gas, often found in the vicinity of outflows (e.g. Garay et al. 2002). In the case of $\mathrm{HC}_{3} \mathrm{~N}$, a hot core tracer, the positive linear correlation is likely suggesting that when there is a larger volume of hot and dense material (and so a higher $\mathrm{HC}_{3} \mathrm{~N}$ integrated intensity), there is also a larger volume of gas contributing to the maser emission. Alternatively, recent work by Taniguchi et al. (2018) suggests that $\mathrm{HC}_{3} \mathrm{~N}$ can trace shocked gas, so the tight correlation might also be reflecting a similar origin, as with the $\mathrm{SiO}$ emission.

Some molecular line ratios have been shown to change with the evolution of the associated high-mass star formation region (e.g. Sanhueza et al. 2012; Hoq et al. 2013). Rathborne et al. (2016) used the MALT90 sample to show that the $\mathrm{HCO}^{+}$to $\mathrm{HNC}$ and $\mathrm{HCN}$ to $\mathrm{HNC}$ integrated intensity ratios increased with evolutionary stage, agreeing with Hoq et al. (2013) that this is probably a reflection of the fact that $\mathrm{HNC}$ is more abundant in less evolved clumps. Rathborne et al. (2016) also found a similar trend in the $\mathrm{HCO}^{+}$to $\mathrm{H}^{13} \mathrm{CO}^{+}$and $\mathrm{HNC}$ to $\mathrm{HN}^{13} \mathrm{C}$ integrated intensity ratios and suggested that this is likely because there is either less selfabsorption or a decrease in optical depth with clump evolution. The 


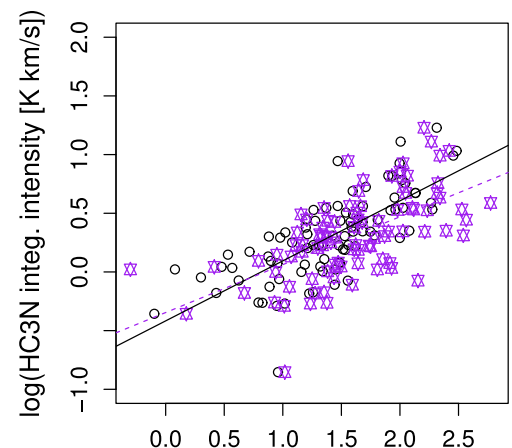

$\log$ (Maser integ. flux [Jy km/s])

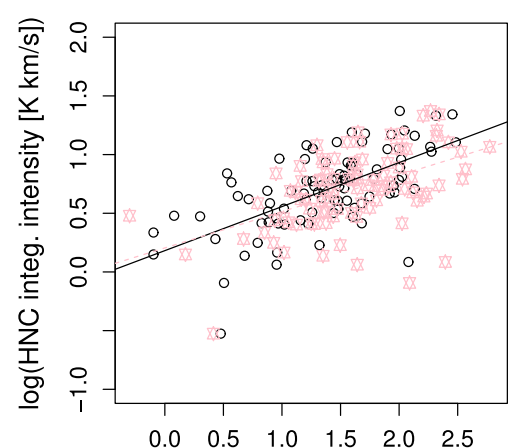

$\log$ (Maser integ. flux [Jy km/s])

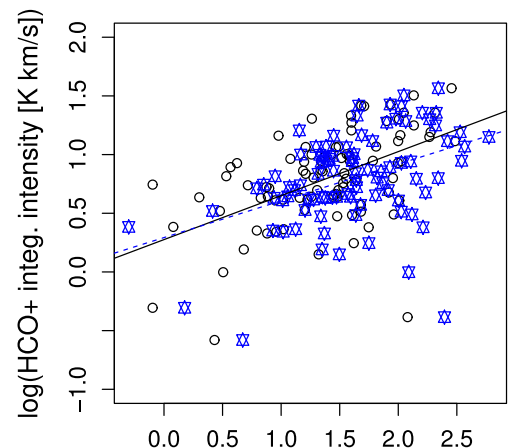

$\log ($ Maser integ. flux [Jy km/s])
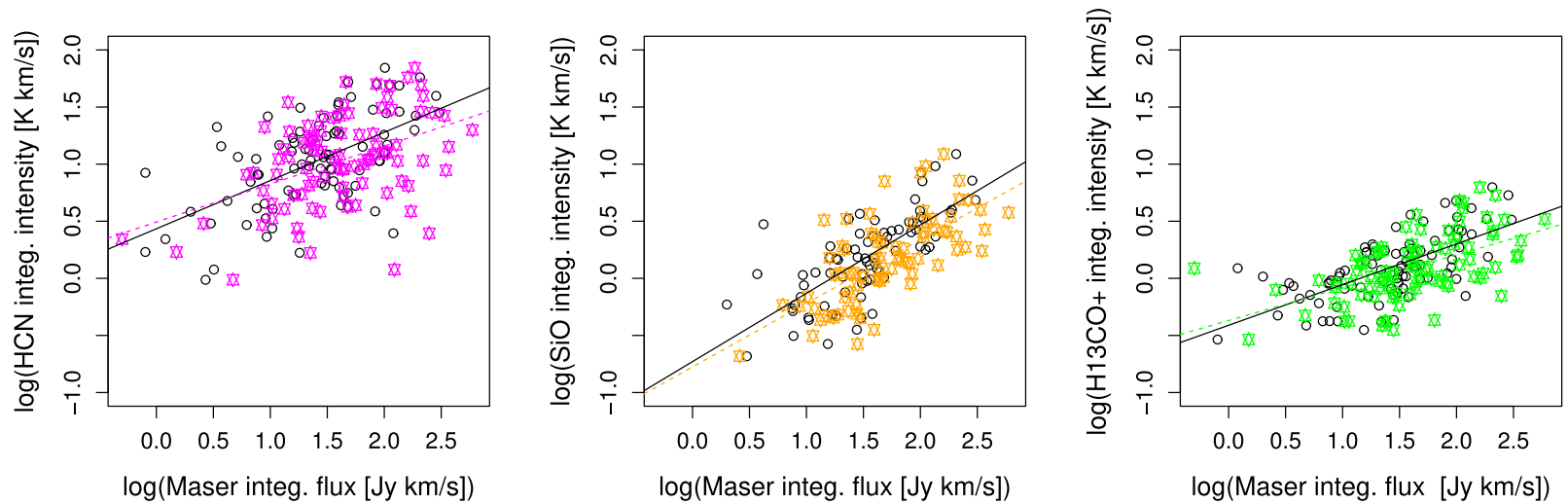

Figure 9. 36- $\mathrm{GHz}$ (coloured stars) and $84-\mathrm{GHz}$ (black circles) methanol maser integrated flux density versus the integrated $\mathrm{HC}_{3} \mathrm{~N}, \mathrm{HNC}, \mathrm{HCO}+, \mathrm{HCN}, \mathrm{SiO}$, and $\mathrm{H}^{13} \mathrm{CO}^{+}$intensities. The lines of best fit are given in each case by a black line for the 84-GHz maser line and a matching coloured dashed line for the 36-GHz methanol masers. The Pearson correlation coefficients between each of the thermal and 36-GHz transition are $0.61,0.50,0.42,0.45,0.69,0.54$, and $0.74,0.63,0.52,0.58,0.73,0.66$ for the $84-\mathrm{GHz}$ transition.

median values of our $\mathrm{HCO}^{+}$to $\mathrm{HNC}, \mathrm{HCN}$ to $\mathrm{HNC}$, and $\mathrm{HCO}^{+}$to $\mathrm{H}^{13} \mathrm{CO}^{+}$integrated line intensities are $1.3,2.1$, and 5.5 , respectively. In their fig. 20, Rathborne et al. (2016) show the median values for these line ratios in the categories of quiescent, protostellar, and $\mathrm{H}$ II regions (since they were looking for evolutionary trends). Our median line ratios are similar to those found in the quiescent $\mathrm{HII}$ regions and protostellar for the $\mathrm{HCO}^{+}$to $\mathrm{HNC}, \mathrm{HCN}$ to $\mathrm{HNC}$, and $\mathrm{HCO}^{+}$to $\mathrm{H}^{13} \mathrm{CO}^{+}$line ratios, respectively. This apparent discrepancy is probably a reflection of our smaller sample and the fact that median values are not a robust indicator of a distribution.

An investigation of the 36- and 84-GHz methanol maser properties with the ratios of $\mathrm{HCO}^{+}$to $\mathrm{HNC}, \mathrm{HCN}$ to $\mathrm{HNC}$, and $\mathrm{HCO}^{+}$to $\mathrm{H}^{13} \mathrm{CO}^{+}$revealed no obvious trends. Even though both our data and that from MALT90 suffer from confusion (given the large Mopra beam) it is possible that our much smaller sample prevents us from revealing a statistical change. Fig. 10 shows the $\mathrm{HCN}$ to $\mathrm{HNC}$ ratio plotted against the $\mathrm{HCO}^{+}$to $\mathrm{H}^{13} \mathrm{CO}^{+}$ratio, revealing a weak positive correlation between the data points. Those targets where radio recombination lines are also detected are scattered throughout Fig. 10, suggesting that the line ratios from our sample are not a good indication of evolution.

Aside from the common molecular lines that were also observed by MALT90, we also observed two thermal methanol transitions at $89.5 \mathrm{GHz}\left(\mathrm{CH}_{3} \mathrm{OH} E\right)$ and $88.9 \mathrm{GHz}\left(\mathrm{CH}_{3} \mathrm{OH} A^{-}\right)$. Their detection rates were relatively low (as can be seen in Fig. 4) with both

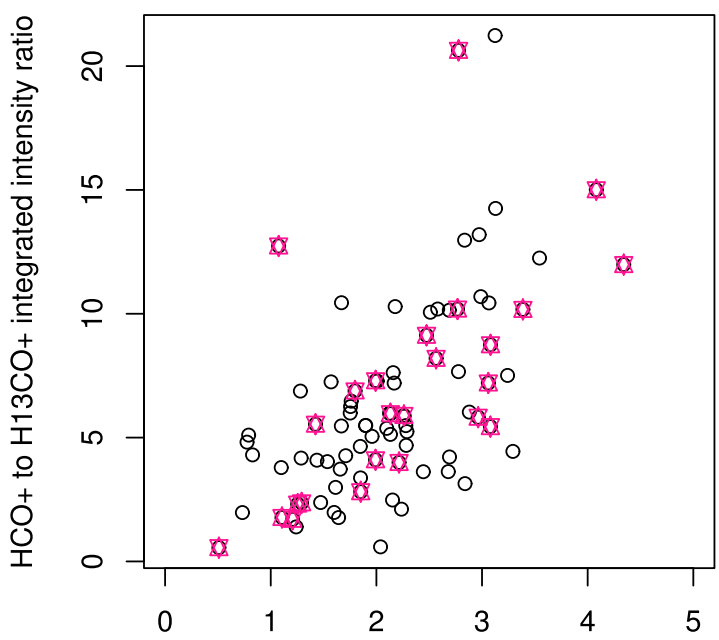

$\mathrm{HCN}$ to HNC integrated intensity ratio

Figure 10. $\mathrm{HCN}$ to $\mathrm{HNC}$ versus $\mathrm{HCO}^{+}$to $\mathrm{H}^{13} \mathrm{CO}^{+}$integrated intensity ratios (black circles). Sources where radio recombination lines are also detected are plotted by pink stars. Note that the $x$-axis has been truncated, excluding two sources with more extreme $\mathrm{HCN}$ to $\mathrm{HNC}$ ratios.

transitions detected towards eight sources and a further source detected in just the $88.9 \mathrm{GHz}$ transition, but with a peak $T_{A}^{*}$ of 
just $0.04 \mathrm{~K}$, below the $3 \sigma$ detection limit of the $89.5 \mathrm{GHz}$ transition. The upper energy level is $171 \mathrm{~K}$ for the $89.5 \mathrm{GHz}$ transition and $328 \mathrm{~K}$ for the $88.9 \mathrm{GHz}$ transition. The ratio of the 89.5 to $88.9 \mathrm{GHz}$ line integrated intensity falls between 0.76 and 1.3 for seven of the eight sources and is 7.1 for G 327.29-0.58, the final source with detections in both transitions. For optically thin methanol gas in local thermodynamic equilibrium, these ratios imply rotational temperatures of $\sim 200 \mathrm{~K}$ for most of the sources and $\sim 70 \mathrm{~K}$ for G 327.29-0.58. Of the nine sources detected at either transition, five are associated with radio recombination lines.

\subsection{Comparison of the class I methanol masers with recombination lines}

Compared to the MALT90 sample of dense clumps, we detected a larger fraction of sources associated with radio recombination lines (29.8 compared with 0.6 per cent) towards our sample of class I methanol masers. This difference can be fully explained by the fact that not only is our data more sensitive to $\mathrm{H} 41 \alpha$ emission (the recombination line included in the MALT90 observations) but we also observe a number of $7 \mathrm{~mm}$ recombination lines (see Table 1). In fact, if we consider only the $\mathrm{H} 41 \alpha$ line and restrict our detection limit to the MALT90 95 per cent completeness level for peak $T_{A}^{*}$, as shown in Fig. 4, the detection limits are consistent, indicating that there is no obvious bias towards the class I methanol maser targets being more evolved than a large fraction of the MALT90 sample.

Of the 28 sites of radio recombination line emission that we detect, many are associated with previously identified hyper and ultracompact H II regions (e.g. Martín-Hernández et al. 2003; Sewilo et al. 2004; Murphy et al. 2010 ). Murphy et al. (2010) summarized the quantitative criteria for the discrimination between hyper and ultracompact $\mathrm{H}$ II regions from the literature. They suggest that the consensus is that recombination line FWHM linewidths less than $40 \mathrm{~km} \mathrm{~s}^{-1}$ are a good indicator of ultracompact $\mathrm{H}$ II regions, while FWHM linewidths greater than $40 \mathrm{~km} \mathrm{~s}^{-1}$ may suggest the presence of an hypercompact $\mathrm{H}$ II region. Using this criterion, only three (G 301.14-0.23, G 5.89-0.39, and G 34.26+0.15) of the 28 recombination lines are associated with hypercompact $\mathrm{H}$ II regions, and the bulk of the detections (25/28) are associated with ultracompact $\mathrm{H}$ II regions.

\subsection{7-, 38.3-, and 38.5-GHz sources}

In the 37.7-, 38.3-, and 38.5-GHz class II methanol maser transitions, we detected 7, 3, and 2 known sources, respectively (Haschick et al. 1989; Ellingsen et al. 2011, 2013, 2018), plus five candidate lines towards four additional sources. As discussed in Section 3.5, the velocities of the candidate sources often show corresponding emission in the 36-GHz transition, lending some credibility to their authenticity. Ellingsen et al. (2018) compared the velocities of 37.7$\mathrm{GHz}$ maser emission with those of the associated 6.7-GHz emission, finding that in the majority of cases, the $37.7-\mathrm{GHz}$ emission was blueshifted with respect to the majority of the accompanying 6.7$\mathrm{GHz}$ emission. Of the four targets where we detect maser candidates in the 37.7-, 38.3-, and 38.5-GHz lines, all of them have velocities that are blueshifted with respect to the $6.7-\mathrm{GHz}$ methanol maser peak (reported in the MMB survey; Green et al. 2010; Caswell et al. 2011; Green et al. 2012). Ellingsen et al. (2013) looked at the temporal variability of 37.7-GHz methanol masers, comparing their 2012 data to data taken in 2009 by Ellingsen et al. (2011). Over the three-year period they found that the largest changes in flux density were at the 40 per cent level. We have observed seven 37.7 $\mathrm{GHz}$ methanol masers that were included in Ellingsen et al. (2013) observations and find that, in the six years between the observations, five of the sources have shown variations at the 15-41 per cent level (we see both increases and reductions at this level, so it is not a calibration problem). The other two sources, G 323.74-0.26 and G $351.42+0.65$, have increased by 122 percent and decreased by 52 per cent in that time.

We further find that $37.7-\mathrm{GHz}$ sources with counterparts at $38.3 \mathrm{GHz}$ and $38.5 \mathrm{GHz}$ do not necessarily show a similar level of variability in all of the detected transitions. In the case of $\mathrm{G} 335.79+0.17$, the $38.3-\mathrm{GHz}$ transition has shown a reduction in peak flux density at approximately the same levels as for the 37.7$\mathrm{GHz}$ transition (41 and 33 percent, respectively). However, for G 345.01+1.79 and G 351.42+0.65, where we detect emission in all three of the lines, we see an increase of 41, 219, and 198 per cent in G $345.01+1.79$ and an increase of 52 per cent and decrease of 31 per cent and 22 per cent in G 351.42+0.65 for the 37.7-, 38.3-, and 38.5-GHz transitions.

\subsection{6- and 86.9-GHz sources}

To date only three sites of 86.6- and 86.9-GHz maser emission have ever been reported [towards G $345.010+1.792$, W3(OH), and W51-IRS1; Cragg et al. 2001; Sutton et al. 2001; Minier \& Booth 2002; Ellingsen et al. 2003). Although the searches that uncovered these sources were not particularly extensive [Ellingsen et al. (2003) targeted 18 sources, Cragg et al. (2001) targeted 17 sources, Minier \& Booth (2002) targeted 23 sources, and Sutton et al. (2001) only targeted $\mathrm{W} 3(\mathrm{OH})]$ masers at $86-$ and $86.9-\mathrm{GHz}$ are expected to be rare given that they are the next transitions up the ladder from the 38.3- and 38.5-GHz transitions (the 38-GHz transitions are $6_{2} \rightarrow 5_{3} A^{-}$and $6_{2} \rightarrow 5_{3} A^{+}$and the $86-\mathrm{GHz}$ transitions are $7_{2}$ $\rightarrow 6_{3} A^{-}$and $7_{2} \rightarrow 6_{3} A^{+}$). Alongside the maser detections, these searches have uncovered a handful of thermal sources detected at either of these transitions (Orion KL, NGC 6334F, G351.77-0.54, W51E2, and NGC 7538-IRS1; Cragg et al. 2001; Minier \& Booth 2002; Ellingsen et al. 2003). Ellingsen et al. (2003) also report the detection of marginal detections towards G 323.740-0.263 and G 339.884-1.259.

Our observations have uncovered nine detections of these transitions, four of which show no deviation from a typically thermal profile shape (G 327.29-0.58, G 351.77-0.54, G 34.26+0.15, W51E1). A further three show possible narrow spectral features in one of the transitions (G339.88-1.26, G344.23-0.57, $\mathrm{G} 351.42+0.65$ ) but the noise levels in the current observations make it difficult to confirm if these are masers. We note that one of these (G $351.42+0.65$ or NGC $6334 \mathrm{~F})$ has also been reported as a thermal source previously. The detection of G339.88-1.26 is very marginal at $86.6 \mathrm{GHz}$, but it is more convincing in the 86.9 $\mathrm{GHz}$ spectrum, allowing us to confirm the marginal detection from Ellingsen et al. (2003).

We have detected two sources with convincing narrow features: $\mathrm{G} 345.01+1.79$ and G 29.96-0.02, the latter of which is a new detection. Interestingly, G 29.96-0.02 shows a different dominant spectral feature at the two frequencies, although a hint of emission is seen in the $86.6-\mathrm{GHz}$ spectrum at the velocity of the $86.9-\mathrm{GHz}$ detection. Higher signal-to-noise observations will be needed in order to show if the spectra are genuinely different.

Interestingly, of the nine sources detected in the 86.6- and 86.9-GHz methanol transitions, six were also detected in the 89.5 and $88.9 \mathrm{GHz}$ lines. The three that have no associated 89.5 - and 
88.9-GHz emission are the most convincing maser detections: G 339.88-1.26, G 345.01+0.1.79, and G 29.96-0.02. Five of the 86.6- and 86.9-GHz detections also show detections in one or more of the 37.7-, 38.3-, and 38.5-GHz methanol transitions. Since the 86.6- and $86.9-\mathrm{GHz}$ are the next transitions up the ladder from the 38.3- and 38.5-GHz transitions, we might expect that masers seen in the 38.3- and $38.5-\mathrm{GHz}$ lines are good targets for 86.6- and 86.9-GHz masers.

\section{SUMMARY}

We have surveyed a sample of 94 class I methanol maser sources (Kurtz et al. 2004; Voronkov et al. 2014) for the little studied 84$\mathrm{GHz}$ class I methanol maser transition. We also conducted nearsimultaneous observations of the $36-\mathrm{GHz}$ class I methanol maser transition to allow meaningful comparison of the two transitions and to derive line ratios. Alongside these observations, the flexibility of the Mopra spectrometer allowed us to concurrently search the sources for the rarer class II methanol maser transitions at 37.7-, 38.3-, 38.5-, 86.6-, and 86.9-GHz as well as a number of thermal molecular and radio recombination lines.

Towards the 94 class I methanol maser targets, we detected 84-GHz emission in 93 (all sources except Mol77) sources and accompanying 36-GHz emission towards 92 sources (all sources except Mol77 and G 45.07+0.13). The spectral profiles of the two transitions are strikingly similar and we use this as the basis for an argument that our sources are likely to contain maser emission even in the case where the spectra are reminiscent of more typically thermal line profiles (since we know the $36-\mathrm{GHz}$ transition shows maser emission from previous interferometric observations). The mean and median peak flux density $36-$ to $84-\mathrm{GHz}$ ratios are 2.4 and 1.6, similar to the integrated flux density mean and median ratios of 2.6 and 1.4. We further find that the stronger $36-\mathrm{GHz}$ masers have higher $36-$ to $84-\mathrm{GHz}$ ratios than the strong $84-\mathrm{GHz}$ sources (as well as the full sample of sources).

We detect one new source of 86.6- and 86.9-GHz methanol maser emission, adding to the small number of masers that have been found in this transition. We detect a further known maser at 86.6- and 86.9-GHz, three sources that may contain narrow maser features, and four sources that show no deviation from a thermal profile. In the 37.7-, 38.3-, and 38.5-GHz transitions, we detect emission (in one or more of the lines) from seven known sources and present four further maser candidates that require follow-up observations with higher sensitivity.

Comparison of the detection rates of thermal molecular lines towards our class I methanol masers with those found towards dense dust clumps across the Galaxy in MALT90 shows almost identical rates in $\mathrm{HNC}, \mathrm{HCN}, \mathrm{HCO}^{+}, \mathrm{H}^{13} \mathrm{CO}^{+}, \mathrm{SiO}, \mathrm{CH}_{3} \mathrm{CN}$, and the $\mathrm{H} 41 \alpha$ recombination line (once the respective detection limits are accounted for). We, however, detect much higher rates of $\mathrm{HC}_{3} \mathrm{~N}$, which we believe indicates that a larger proportion of the class I maser target list are warm protostellar sources compared to the MALT90 sample.

We find a close correspondence between the peak velocity of the class I maser sources and the thermal line counterparts, in particular with $\mathrm{HNC}, \mathrm{HCO}^{+}$, and $\mathrm{H}^{13} \mathrm{CO}^{+}$, supporting a result found previously for $44-\mathrm{GHz}$ methanol masers that class I methanol masers are generally excellent tracers of systemic velocities.

There is a positive correlation between the 36- and $84-\mathrm{GHz}$ integrated flux densities and integrated intensities of the detected thermal lines. Given the similarity of the slopes in each of the relationships, we suggest that this indicates that the maser integrated flux density is a reflection of the available quantity of molecular gas.

\section{ACKNOWLEDGEMENTS}

The Mopra radio telescope is part of the Australia Telescope National Facility. Operations support was provided by the University of New South Wales, the University of Adelaide, The University of Sydney, The University of Newcastle, Nagoya University, NASA Goddard Space Flight Centre and Western Sydney University. This research has made use of: NASA's Astrophysics Data System Abstract Service; and the SIMBAD data base, operated at CDS, Strasbourg, France. J.R.D. acknowledges the support of an Australian Research Council (ARC) DECRA Fellowship (project number DE170101086). S.P.E. acknowledges the support of ARC Discovery Project (project number DP180101061).

\section{REFERENCES}

Batrla W., Menten K. M., 1988, ApJ, 329, 117

Batrla W., Matthews H. E., Menten K. M., Walmsley C. M., 1987, Nature, 326,49

Breen S. L., Ellingsen S. P., Caswell J. L., Lewis B. J., 2010, MNRAS, 401, 2219

Breen S. L., Ellingsen S. P., Caswell J. L., Green J. A., Voronkov M. A., Fuller G. A., Quinn L. J., Avison A., 2012a, MNRAS, 421, 1703

Breen S. L., Ellingsen S. P., Caswell J. L., Green J. A., Voronkov M. A., Fuller G. A., Quinn L. J., Avison A., 2012b, MNRAS, 426, 2189

Breen S. L., Ellingsen S. P., Contreras Y., Green J. A., Caswell J. L., Stevens J. B., Dawson J. R., Voronkov M. A., 2013, MNRAS, 435, 524

Breen S. L. et al., 2014, MNRAS, 438, 3368

Breen S. L. et al., 2015, MNRAS, 450, 4109

Breen S. L., Ellingsen S. P., Caswell J. L., Green J. A., Voronkov M. A., Avison A., Fuller G. A., Quinn L.J ., 2016, MNRAS, 459, 4066

Caswell J. L. et al., 2010, MNRAS, 404, 1029

Caswell J. L. et al., 2011, MNRAS, 417, 1964

Caswell J. L., Yi J., Booth R. S., Cragg D. M., 2000, MNRAS, 313, 599

Chen X., Ellingsen S. P., Shen Z.-Q., Titmarsh A., Gan C.-G., 2011, ApJS, 196,9

Cragg D. M., Sobolev A. M., Ellingsen S. P., Caswell J. L., Godfrey P. D., Salii S. V., Dodson R. G., 2001, MNRAS, 323, 939

Cragg D. M., Sobolev A. M., Caswell J. L., Ellingsen S. P., Godfrey P. D., 2004, MNRAS, 351, 1327

Cragg D. M., Sobolev A. M., Godfrey P. D., 2005, MNRAS, 360, 533

Cyganowski C. J., Brogan C. L., Hunter T. R., Churchwell E., 2009, ApJ, 702,1615

Ellingsen S. P., 2005, MNRAS, 359, 1498

Ellingsen S. P., Cragg D. M., Minier V., Muller E., Godfrey P. D., 2003, MNRAS, 344, 73

Ellingsen S. P., Cragg D. M., Lovell J. E. J., Sobolev A. M., Ramsdale P. D., Godfrey P. D., 2004, MNRAS, 354, 401

Ellingsen S. P., Breen S. L., Sobolev A. M., Voronkov M. A., Caswell J. L., Lo N., 2011, ApJ, 742, 109

Ellingsen S. P., Breen S. L., Voronkov M. A., Dawson J. R., 2013, MNRAS, 429, 3501

Ellingsen S. P., Chen X., Breen S. L., Qiao H.-H., 2017, MNRAS, 472, 604

Ellingsen S. P., Voronkov M. A., Breen S. L., Caswell J. L., Sobolev A., 2018, MNRAS, 480, 4851

Garay G., Mardones D., Rodríguez L. F., Caselli P., Bourke T. L., 2002, ApJ, 567, 980

Gómez-Ruiz A. I., Kurtz S. E., Araya E. D., Hofner P., Loinard L., 2016, ApJS, 222, 18

Green J. A. et al., 2010, MNRAS, 409, 913

Green J. A. et al., 2012, MNRAS, 420, 3108

Green J. A. et al., 2017, MNRAS, 469, 1383 
Guzmán A. E., Sanhueza P., Contreras Y., Smith H. A., Jackson J. M., Hoq S., Rathborne J. M., 2015, ApJ, 815, 130

Haschick A. D., Baan W. A., Menten K. M., 1989, ApJ, 346, 330

Hoq S. et al., 2013, ApJ, 777, 157

Jordan C. H. et al., 2015, MNRAS, 448, 2344

Jordan C. H., Walsh A. J., Breen S. L., Ellingsen S. P., Voronkov M. A., Hyland L. J., 2017, MNRAS, 471, 3915

Kalenskii S. V., Slysh V. I., Val'tts I. E., Winnberg A., Johansson L. E. B., 2001, Astron. Rep., 45, 26

Kalenskii S. V., Promyslov V. G., Slysh V. I., Bergman P., Winnberg A., 2006, Astron. Rep., 50, 289

Kalenskii S. V., Johansson L. E. B., Bergman P., Kurtz S., Hofner P., Walmsley C. M., Slysh V. I., 2010, MNRAS, 405, 613

Kim C.-H., Kim K.-T., Park Y.-S., 2018, ApJS, 236, 31

Kurtz S., Hofner P., Álvarez C. V., 2004, ApJS, 155, 149

Ladd N., Purcell C., Wong T., Robertson S., 2005, PASA, 22, 62

Leurini S., Menten K. M., Walmsley C. M., 2016, A\&A, 592, 31

Lilley A. E., Palmer P., 1968, ApJS, 16, 143

Martín-Hernández N. L., Bik A., Kaper L., Tielens A. G. G. M., Hanson M. M., 2003, A\&A, 405, 175

McCarthy T. P., Ellingsen S. P., Chen X., Breen S. L., Henkel C., Voronkov M. A., Qiao H.-H., 2017, ApJ, 846, 156

McCarthy T. P., Ellingsen S. P., Voronkov M. A., Cimò G., 2018a, MNRAS, 477, 507

McCarthy T. P., Ellingsen S. P., Breen S. L., Henkel C., Voronkov M. A., 2018b, MNRAS, 480, 4578

McEwen B. C., Pihlström Y. M., Sjouwerman L. O., 2014, ApJ, 793, 133

Menten K. M., 1991a, in Haschick A., Ho P. T. P., eds, ASP Conf. Ser. Vol. 16, Atoms, Ions and Molecules: New Results in Spectral Line Astrophysics. Astron. Soc. Pac. San Francisco, p. 119

Menten K. M., 1991b, ApJ, 380, L75

Minier V., Booth R. S., 2002, A\&A, 387, 179

Minier V., Ellingsen S. P., Norris R. P., Booth R. S., 2003, A\&A, 403, 1095

Müller H. S. P., Menten K. M., Mäder H., 2004, A\&A, 428, 1019

Murphy T., Cohen M., Ekers R. D., Green A. J., Wark R. M., Moss V., 2010, MNRAS, 405, 1560

Pratap P., Shute P. A., Keane T. C., Battersby C., Sterling S., 2008, AJ, 135, 1718

Rathborne J. M. et al., 2016, PASA, 33, 30

Remijan A. J., Markwick-Kemper A., ALMA Working Group on Spectral Line Frequencies, 2007, BAAS, 39, 963

Rodríguez-Garza C. B., Kurtz S. E., Gómez-Ruiz A. I., Hofner P., Araya E. D., Kalenskii S. V., 2017, ApJS, 233, 4

Rodríguez-Garza C. B., Kurtz S. E., Gómez-Ruiz A. I., Hofner P., Araya E. D., Kalenskii S. V., 2018, in Tarchi A., Reid M. J., Castangia P., eds, Proc. IAU Symp. 336, Astrophysical Masers: Unlocking the Mysteries of the Universe. Cambridge Univ. Press, Cambridge, p. 239
Salii S. V., Sobolev A. M., Kalinina N. D., 2002, Astron. Rep., 46, 955

Sanhueza P., Jackson J. M., Foster J. B., Garay G., Silva A., Finn S. C., 2012, ApJ, 756, 60

Sewilo M., Churchwell E., Kurtz S., Goss W. M., Hofner P., 2004, ApJ, 605, 285

Slysh V. I., Kalenskii S. V., Val'tts I. E., Otrupcek R., 1994, MNRAS, 268, 464

Sutton E. C., Sobolev A. M., Ellingsen S. P., Cragg D. M., Mehringer D. M., Ostrovskii A. B., Godfrey P. D., 2001, ApJ, 554, 173

Taniguchi K., Miyamoto Y., Saito M., Sanhueza P., Shimoikura T., Dobashi K., Nakamura F., Ozeki H., 2018, ApJ, 866, 32

Umemoto T., Mochizuki N., Shibata K. M., Roh D.-G., Chung H.-S., 2007, in Chapman J. M., Baan W. A., eds, Proc. IAU Symp. 242, Astrophysical Masers and their Environments, Cambridge Univ. Press, Cambridge, p. 234

Urquhart J. S. et al., 2010, PASA, 27, 321

Val'tts I. E., Ellingsen S. P., Slysh V. I., Kalenskii S. V., Otrupcek R., Voronkov M. A., 1999, MNRAS, 310, 1077

Val'tts I. E., Ellingsen S. P., Slysh V. I., Kalenskii S. V., Otrupcek R., Larionov G. M., 2000, MNRAS, 317, 315

Voronkov M. A., Brooks K. J., Sobolev A. M., Ellingsen S. P., Ostrovskii A. B., Caswell J. L., 2006, MNRAS, 373, 411

Voronkov M. A., Walsh A. J., Caswell J. L., Ellingsen S. P., Breen S. L., Longmore S. N., Purcell C. R., Urquhart J. S., 2011, MNRAS, 413, 2339

Voronkov M. A., Caswell J. L., Ellingsen S. P., Green J. A., Breen S. L., 2014, MNRAS, 439, 2584

Xu Y., Li J. J., Hachisuka K., Pandian J. D., Menten K. M., Henkel C., 2008, A\&A, 485, 729

Xu L.-H., Lovas F. J., 1997, J. Phys. Chem. Ref. Data, 26, 17

Yusef-Zadeh F., Cotton W., Viti S., Wardle M., Royster M., 2013, ApJ, 764, L19

\section{SUPPORTING INFORMATION}

Supplementary data are available at MNRAS online.

Table A1. Molecular and recombination line Gaussian fit parameters.

Please note: Oxford University Press is not responsible for the content or functionality of any supporting materials supplied by the authors. Any queries (other than missing material) should be directed to the corresponding author for the article.

This paper has been typeset from a $\mathrm{T}_{\mathrm{E}} \mathrm{X} / \mathrm{L} \mathrm{T} \mathrm{E} \mathrm{X}$ file prepared by the author. 